\title{
Higher Order Asymptotic Expansion for the Heat Equation with a Nonlinear Boundary Condition
}

By

\author{
Tatsuki KAWAKAMI
}

(Osaka Prefecture University, Japan)

\begin{abstract}
We consider the heat equation with a nonlinear boundary condition: $(P)$ $\partial_{t} u=\Delta u$ in $\boldsymbol{R}_{+}^{N} \times(0, \infty), \partial_{v} u=\kappa|u|^{p-1} u$ on $\partial \boldsymbol{R}_{+}^{N} \times(0, \infty), u(x, 0)=\varphi(x)$ in $\boldsymbol{R}_{+}^{N}$, where $\boldsymbol{R}_{+}^{N}=\left\{x=\left(x^{\prime}, x_{N}\right) \in \boldsymbol{R}^{N}: x_{N}>0\right\}, N \geq 2, \partial_{t}=\partial / \partial t, \partial_{v}=-\partial / \partial x_{N}, \kappa \in \boldsymbol{R}$, and $p>1+1 / N$. Let $u$ be a solution of $(\mathrm{P})$ satisfying $\sup _{t>0}(1+t)^{(N / 2)(1-1 / q)}\left[\|u(t)\|_{L^{q}\left(\boldsymbol{R}_{+}^{N}\right)}\right.$ $\left.+t^{1 /(2 q)}\|u(t)\|_{L^{q\left(\partial \boldsymbol{R}_{+}^{N}\right)}}\right]<\infty, q \in[1, \infty]$. In this paper, under suitable assumptions of the initial function $\varphi$, we establish the method of obtaining higher order asymptotic expansions of the solution $u$ as $t \rightarrow \infty$.

Key Words and Phrases. Nonlinear boundary condition, Asymptotic expansion, Large time behavior.

2010 Mathematics Subject Classification Numbers. 35B40, 35K61.
\end{abstract}

\section{Introduction}

In this paper we consider the large time behavior of the solution of the heat equation in the half space $\boldsymbol{R}_{+}^{N}=\left\{x=\left(x^{\prime}, x_{N}\right) \in \boldsymbol{R}^{N}: x_{N}>0\right\}$ with a nonlinear boundary condition,

$$
\begin{cases}\partial_{t} u=\Delta u & \text { in } \boldsymbol{R}_{+}^{N} \times(0, \infty), \\ \partial_{v} u=\kappa|u|^{p-1} u & \text { on } \partial \boldsymbol{R}_{+}^{N} \times(0, \infty), \\ u(x, 0)=\varphi(x) & \text { in } \boldsymbol{R}_{+}^{N},\end{cases}
$$

where $N \geq 2, \partial_{t}=\partial / \partial t, \partial_{v}=-\partial / \partial x_{N}, p>1$, and

$$
\varphi \in X_{K}\left(\boldsymbol{R}_{+}^{N}\right):=L^{\infty}\left(\boldsymbol{R}_{+}^{N}\right) \cap\left\{f \in L^{1}\left(\boldsymbol{R}_{+}^{N}\right): \int_{\boldsymbol{R}_{+}^{N}}(1+|x|)^{K}|f(x)| d x<\infty\right\}
$$

for some $K \geq 0$. Nonlinear boundary value problem (1.1) can be physically interpreted as a nonlinear radiation law, and has been studied in many papers (see [1]-[5], [7], [10]-[12], and references therein). For the case $\kappa>0, \varphi \geq 0$, and $\varphi \not \equiv 0$, it is well known that if $1<p \leq 1+1 / N$, then the problem (1.1) does not have any positive global in time solutions, and if $p>1+1 / N$, then, for some initial datum $\varphi$, the problem (1.1) has a positive global in time solution (see, for example, [3] and [5]). In particular, for the case $p>1+1 / N$, in [10], 
the author of this paper proved that, if the initial datum $\varphi$ satisfies (1.2) with $K=0$ and $\|\varphi\|_{L^{1}\left(\boldsymbol{R}_{+}^{N}\right)}\|\varphi\|_{L^{\infty}\left(\boldsymbol{R}_{+}^{N}\right)}^{N(p-1)-1}$ is sufficiently small, then the solution $u$ of (1.1) exists globally in time, and satisfies

$$
\sup _{t>0}(1+t)^{(N / 2)(1-1 / q)}\left[\|u(t)\|_{L^{q}\left(\boldsymbol{R}_{+}^{N}\right)}+t^{1 /(2 q)}\|u(t)\|_{L^{q}\left(\partial \boldsymbol{R}_{+}^{N}\right)}\right]<\infty, \quad q \in[1, \infty] .
$$

Furthermore he proved that, if the solution $u$ satisfies (1.3), then it holds that

$$
\left\{\begin{array}{l}
\int_{\boldsymbol{R}_{+}^{N}} u(x, t) d x \text { converges to a constant } M \text { as } t \rightarrow \infty \text { and } \\
\lim _{t \rightarrow \infty}\|u(\cdot, t)-M \Gamma(\cdot, 0,1+t)\|_{L^{q}\left(\boldsymbol{R}_{+}^{N}\right)} /\|\Gamma(\cdot, 0,1+t)\|_{L^{q}\left(\boldsymbol{R}_{+}^{N}\right)}=0 \\
\quad \text { for any } q \in[1, \infty] .
\end{array}\right.
$$

Here $\Gamma=\Gamma(x, y, t)$ is the Green function for the heat equation on $\boldsymbol{R}_{+}^{N}$ with the homogeneous Neumann boundary condition, that is,

$$
\Gamma(x, y, t)=G_{N}(x-y, t)+G_{N}\left(x-y_{*}, t\right), \quad x, y \in \boldsymbol{R}_{+}^{N}, t>0,
$$

where $y_{*}=\left(y^{\prime},-y_{N}\right)$ for $y=\left(y^{\prime}, y_{N}\right) \in \boldsymbol{R}_{+}^{N}$ and $G_{N}$ is the $N$-dimensional Gauss kernel, that is,

$$
G_{N}(x, t)=(4 \pi t)^{-N / 2} \exp \left(-\frac{|x|^{2}}{4 t}\right) .
$$

On the other hand, under condition (1.2) with $K=0$, if $\kappa=0$, then we can easily obtain (1.3) and (1.4), and if $\kappa<0$ with $p>1+1 / N$, then, applying the comparison principle and modifying the argument in [10], we see that there exists a solution $u$ satisfying (1.3) and (1.4).

Assertion (1.4) gives the large time behavior, in particular the first term of asymptotic expansions, of the solution $u$ of (1.1) satisfying (1.3). The large time behavior of the solutions for nonlinear parabolic problem including the problem (1.1) is influenced by the behavior of the initial datum at the spatial infinity and the nonlinearity, and has been studied in many papers (see [6]-[11], [13], and references therein). In fact, for the problem

$$
\begin{cases}\partial_{t} u=\Delta u & \text { in } \boldsymbol{R}_{+}^{N} \times(0, \infty), \\ \partial_{v} u=u^{p} & \text { on } \partial \boldsymbol{R}_{+}^{N} \times(0, \infty), \\ u(x, 0)=\varphi(x) \geq 0 & \text { in } \boldsymbol{R}_{+}^{N}\end{cases}
$$

with $p>1+1 / N$, in [11], applying the entropy dissipation method, the author of this paper proved that, under condition (1.2) with $K=2$, if the solution $u$ of (1.6) satisfies (1.3), then, for any $q \in[1, \infty]$, 


$$
\begin{aligned}
& t^{(N / 2)(1-1 / q)}\|R(t)\|_{L^{q}\left(\boldsymbol{R}_{+}^{N}\right)} \\
& \quad= \begin{cases}O\left(t^{-1 / 2}\right)+O\left(t^{-(N / 4)(p-1-1 / N)}\right) & \text { if } N(p-1-1 / N) \neq 2, \\
O\left(t^{-1 / 2}(\log t)^{1 / 2}\right) & \text { if } N(p-1-1 / N)=2,\end{cases}
\end{aligned}
$$

as $t \rightarrow \infty$, where

$$
R(x, t)=u(x, t)-M \Gamma(x, 0,1+t), \quad(x, t) \in \boldsymbol{R}_{+}^{N} \times(0, \infty) .
$$

Furthermore, in [7], the author of this paper and Ishige showed that, under condition $\varphi \in L^{\infty}\left(\boldsymbol{R}_{+}^{N}\right) \cap L^{2}\left(\boldsymbol{R}_{+}^{N}, e^{|x|^{2} / 4} d x\right)$ and $(N-2) p<N$, if the solution $u$ of (1.6) satisfies (1.3), then, for any $q \in[1, \infty]$,

$$
t^{(N / 2)(1-1 / q)}\|R(t)\|_{L^{q}\left(\boldsymbol{R}_{+}^{N}\right)}=O\left(t^{-1 / 2}\right)+O\left(t^{-(N / 2)(p-1-1 / N)}\right)
$$

as $t \rightarrow \infty$. These results imply that, if the initial datum $\varphi$ belongs to some suitable spaces like $X_{2}\left(\boldsymbol{R}_{+}^{N}\right)$ or $L^{\infty}\left(\boldsymbol{R}_{+}^{N}\right) \cap L^{2}\left(\boldsymbol{R}_{+}^{N}, e^{|x|^{2} / 4} d x\right)$, then we can obtain more precise estimate on the difference between the solution and its asymptotic profiles than (1.4). However, from these results, we can not obtain the relationship between the decay rate of $\|R(t)\|_{L^{q}\left(\boldsymbol{R}_{+}^{N}\right)}$ and the behavior of the initial datum $\varphi$ at the spatial infinity. Actually, although the initial conditions are different, for sufficiently large $p$, these two inequalities (1.7) and (1.9) yield the same estimate, that is, $t^{(N / 2)(1-1 / q)}\|R(t)\|_{L^{q}\left(\boldsymbol{R}_{+}^{N}\right)}=O\left(t^{-1 / 2}\right)$ as $t \rightarrow \infty$. Furthermore, as far as we know, there are no results treating higher order asymptotic expansions of the solution of (1.1) even if $\varphi \in C_{0}^{\infty}\left(\boldsymbol{R}_{+}^{N}\right)$.

Recently, for the Cauchy problem of the semilinear heat equation

$$
\partial_{t} u=\Delta u+\kappa|u|^{p-1} u \quad \text { in } \boldsymbol{R}^{N} \times(0, \infty), \quad u(x, 0)=\varphi(x) \quad \text { in } \boldsymbol{R}^{N}
$$

with $\kappa \in \boldsymbol{R}$ and $p>1+2 / N$, in [9], improving the argument in [6], the author of this paper and Ishige established the useful method which derives higher order asymptotic expansions of the solutions for the Cauchy problem (1.10). The argument in [9] is applicable to the large class of the nonlinear parabolic equations in the whole space (see [8]). However it is not applicable directly to nonlinear boundary value problem (1.1) because the integral equation has a different kernel and the nonlinear term appears on the boundary (see Definition 2.1). In this paper, as an extension of [9], under condition (1.2) and $p>$ $1+1 / N$, we consider the initial-boundary value problem (1.1), and study the large time behavior of the solutions satisfying (1.3). In particular, modifying the arguments in [8] and [9], we give higher order asymptotic expansions of the solution $u$ of (1.1). Throughout this paper we write

$$
A_{p}:=\frac{N}{2}(p-1)
$$

for simplicity. We recall that $A_{p}>1 / 2$ under condition $p>1+1 / N$. 
The rest of this paper is organized as follows. In Section 2 we give some notation and introduce some operators. Furthermore we state the main results of this paper. In Section 3 we give some preliminaries. Section 4 is devoted to the proof of main results.

\section{Notation and main results}

In this section we give some notation and the definition of the solution of (1.1). Furthermore we introduce some operators $P_{K}(t)$ and $Q_{K}(t)$, and state the main results of this paper.

\subsection{Notation}

We first introduce some notation. Let $N_{0}=N \cup\{0\}$ and $\mathcal{O}$ be the set of odd numbers. For any $k \in \boldsymbol{R}$, let $[k]$ be an integer such that $k-1<[k] \leq k$. For any multi-index $\alpha=\left(\alpha_{1}, \ldots, \alpha_{N}\right) \in N_{0}^{N}$, we put

$$
\begin{aligned}
& |\alpha|:=\sum_{i=1}^{N}\left|\alpha_{i}\right|, \quad \alpha !:=\prod_{i=1}^{N} \alpha_{i} !, \quad x^{\alpha}:=\prod_{i=1}^{N} x_{i}^{\alpha_{i}}, \quad \partial_{x}^{\alpha}:=\frac{\partial^{|\alpha|}}{\partial x_{1}^{\alpha_{1}} \ldots \partial x_{N}^{\alpha_{N}}}, \\
& J(\alpha):=\left\{\rho=\left(\rho_{1}, \ldots, \rho_{N}\right) \in N_{0}^{N} \backslash\{\alpha\}: \rho_{i} \leq \alpha_{i} \text { for all } i=1, \ldots, N\right\}, \\
& g_{\alpha}(x, t):=\frac{(-1)^{|\alpha|}}{\alpha !}\left(\partial_{x}^{\alpha} \Gamma\right)(x, 0,1+t),
\end{aligned}
$$

where $\Gamma(x, y, t)$ is the function given in (1.5). In particular, we write $g(x, t)=g_{0}(x, t)$ for simplicity. Furthermore, for any multi-index $\tilde{\alpha} \in N_{0}^{N-1}$, we put

$$
\tilde{g}_{\tilde{\alpha}}\left(x^{\prime}, t\right):=\frac{(-1)^{|\tilde{\alpha}|}}{\tilde{\alpha} !}\left(\partial_{x^{\prime}}^{\tilde{\alpha}} G_{N-1}\right)\left(x^{\prime}, 1+t\right) .
$$

In particular, we write $\tilde{g}(x, t)=\tilde{g}_{0}(x, t)$ for simplicity. We denote by $S(t) \varphi$ the unique bounded solution of the heat equation on $\boldsymbol{R}_{+}^{N}$ with the homogeneous Neumann boundary condition and the initial datum $\varphi$, that is,

$$
(S(t) \varphi)(x):=\int_{\boldsymbol{R}_{+}^{N}} \Gamma(x, y, t) \varphi(y) d y, \quad x \in \boldsymbol{R}_{+}^{N}, t>0 .
$$

Furthermore we denote by $e^{t \Delta^{\prime}} \psi$ the unique bounded solution of the heat equation on $\boldsymbol{R}^{N-1}$ with the initial datum $\psi$, that is,

$$
\left(e^{t \Delta^{\prime}} \psi\right)\left(x^{\prime}\right):=\int_{\boldsymbol{R}^{N-1}} G_{N-1}\left(x^{\prime}-y^{\prime}, t\right) \psi\left(y^{\prime}\right) d y^{\prime}, \quad x^{\prime} \in \boldsymbol{R}^{N-1}, t>0 .
$$


For any two nonnegative functions $f_{1}$ and $f_{2}$ defined in a subset $D$ of $[0, \infty)$, we say $f_{1}(t) \preceq f_{2}(t)$ for all $t \in D$ if there exists a positive constant $C$ such that $f_{1}(t) \leq C f_{2}(t)$ for all $t \in D$. For any $m \geq 0$, we denote by $L_{m}^{1}$ and $L_{m, \boldsymbol{R}^{N-1}}^{1}$ the function spaces $L^{1}\left(\boldsymbol{R}_{+}^{N},(1+|x|)^{m} d x\right)$ and $L^{1}\left(\boldsymbol{R}^{N-1},\left(1+\left|x^{\prime}\right|\right)^{m} d x^{\prime}\right)$ respectively. In what follows, we write

$$
\begin{aligned}
& \|\cdot\|_{q}=\|\cdot\|_{L^{q}\left(\boldsymbol{R}_{+}^{N}\right)}, \quad\|\| \cdot\left\|_{m}=\right\| \cdot \|_{L^{1}\left(\boldsymbol{R}_{+}^{N},(1+|x|)^{m} d x\right)}, \\
& \|\cdot\|_{q, \partial \boldsymbol{R}_{+}^{N}}=\|\cdot\|_{L^{q}\left(\partial \boldsymbol{R}_{+}^{N}\right)}, \quad\|\| \cdot\|\|_{m, \partial \boldsymbol{R}_{+}^{N}}=\|\cdot\|_{L^{1}\left(\partial \boldsymbol{R}_{+}^{N},(1+|x|)^{m} d \sigma\right)}, \\
& \|\cdot\|_{q, \boldsymbol{R}^{N-1}}=\|\cdot\|_{L^{q}\left(\boldsymbol{R}^{N-1}\right)}, \quad\|\| \cdot\left\|_{m, \boldsymbol{R}^{N-1}}=\right\| \cdot \|_{L^{1}\left(\boldsymbol{R}^{N-1},\left(1+\left|x^{\prime}\right|\right)^{m} d x^{\prime}\right)},
\end{aligned}
$$

for simplicity, where $q \in[1, \infty]$ and $m \geq 0$.

Next we give the definition of the solution of (1.1).

Definition 2.1. Let $\varphi \in X_{0}\left(\boldsymbol{R}_{+}^{N}\right)$. Then the function $u \in C\left(\overline{\boldsymbol{R}_{+}^{N}} \times(0, \infty)\right) \cap$ $L^{\infty}\left(0, \infty: L^{\infty}\left(\boldsymbol{R}_{+}^{N}\right)\right)$ is said to be a solution of (1.1) if

$$
\begin{aligned}
u(x, t)= & \int_{\boldsymbol{R}_{+}^{N}} \Gamma(x, y, t) \varphi(y) d y \\
& +\kappa \int_{0}^{t} \int_{\partial \boldsymbol{R}_{+}^{N}} \Gamma(x, y, t-s)|u(y, s)|^{p-1} u(y, s) d \sigma_{y} d s
\end{aligned}
$$

holds for all $(x, t) \in \boldsymbol{R}_{+}^{N} \times(0, \infty)$. Here $\Gamma$ is the Green function given by (1.5) and $d \sigma_{y}$ is the $(N-1)$-dimensional Lebesgue measure on $\partial \boldsymbol{R}_{+}^{N}=\boldsymbol{R}^{N-1}$.

It is known that, under the above definition, for any initial datum $\varphi \in$ $X_{0}\left(\boldsymbol{R}_{+}^{N}\right)$, the problem (1.1) has a unique classical solution (see, for example, [7]). Furthermore, combining (1.5), and (2.1)-(2.3) we have

$$
u(x, t)=(S(t) \varphi)(x)+2 \kappa \int_{0}^{t} G_{1}\left(x_{N}, t-s\right)\left(e^{(t-s) \Delta^{\prime}} \gamma\left[|u(s)|^{p-1} u(s)\right]\right) d s
$$

for all $(x, t) \in \boldsymbol{R}_{+}^{N} \times(0, \infty)$, where $\gamma[f]\left(x^{\prime}\right)=f\left(x^{\prime}, 0\right)$ for all $x^{\prime} \in \boldsymbol{R}^{N-1}$.

The end of this subsection we introduce some operators $P_{K}(t)$ and $Q_{K}(t)$. Following [6] and [9], for any $k \geq 0$ and $t>0$, we introduce a linear operator $P_{k}(t)$ on $L_{k}^{1}$ by

$$
\left[P_{k}(t) f_{1}\right](x):=f_{1}(x)-\sum_{|\alpha| \leq k, \alpha_{N} \notin \mathcal{O}} M_{\alpha}\left(f_{1}, t\right) g_{\alpha}(x, t),
$$

where $f_{1} \in L_{k}^{1}$ and $M_{\alpha}(f, t)$ is a constant defined inductively (in $\alpha$ ) by

$$
M_{\alpha}\left(f_{1}, t\right):=\int_{\boldsymbol{R}_{+}^{N}} x^{\alpha} f_{1}(x) d x-\sum_{\rho \in J(\alpha), \rho_{N} \notin \mathcal{O}} M_{\rho}\left(f_{1}, t\right) \int_{\boldsymbol{R}_{+}^{N}} x^{\alpha} g_{\rho}(x, t) d x .
$$


Furthermore, for any $k \geq 0$ and $t>0$, we put

$$
\left[Q_{k}(t) f_{2}\right]\left(x^{\prime}\right):=f_{2}\left(x^{\prime}\right)-\sum_{|\tilde{\alpha}| \leq k} \tilde{M}_{\tilde{\alpha}}\left(f_{2}, t\right) \tilde{g}_{\tilde{\alpha}}\left(x^{\prime}, t\right),
$$

where $f_{2} \in L_{k, \boldsymbol{R}^{N-1}}^{1}$ and $\tilde{M}_{\tilde{\alpha}}(f, t)$ is a constant defined inductively (in $\tilde{\alpha}$ ) by

$$
\tilde{M}_{\tilde{\alpha}}\left(f_{2}, t\right):=\int_{\boldsymbol{R}^{N-1}}\left(x^{\prime}\right)^{\tilde{\alpha}} f_{2}\left(x^{\prime}\right) d x^{\prime}-\sum_{\rho \in J(\tilde{\alpha})} \tilde{M}_{\rho}\left(f_{2}, t\right) \int_{\boldsymbol{R}^{N-1}}\left(x^{\prime}\right)^{\tilde{\alpha}} \tilde{g}_{\rho}\left(x^{\prime}, t\right) d x^{\prime}
$$

Then, it is easy to see that the operators $P_{k}(t)$ and $Q_{k}(t)$ satisfy

$$
\begin{aligned}
& \int_{\boldsymbol{R}_{+}^{N}} x^{\alpha}\left[P_{k}(t) f_{1}\right](x) d x=0, \quad|\alpha| \leq k \text { with } \alpha_{N} \notin \mathcal{O}, \\
& \int_{\boldsymbol{R}^{N-1}}\left(x^{\prime}\right)^{\tilde{\alpha}}\left[Q_{k}(t) f_{2}\right]\left(x^{\prime}\right) d x^{\prime}=0, \quad|\tilde{\alpha}| \leq k,
\end{aligned}
$$

for all $t>0$ (see [6]). This operators are key of our proof, in particular (2.8) and (2.9) are crucial properties in our analysis. Furthermore, if $\varphi \in X_{K}\left(\boldsymbol{R}_{+}^{N}\right)$ with $K \geq 0$, then, applying the operator $P_{K}(t)$ to $S(t) \varphi$, we have

$$
\begin{aligned}
P_{K}(t) S(t) \varphi & =S(t) \varphi-\sum_{|\alpha| \leq K, \alpha_{N} \notin \mathcal{U}} M_{\alpha}(S(t) \varphi, t) g_{\alpha}(x, t) \\
& =S(t) \varphi-\sum_{|\alpha| \leq K, \alpha_{N} \notin \mathcal{O}} M_{\alpha}(\varphi, 0) g_{\alpha}(x, t)=S(t)\left[P_{K}(0) \varphi\right]
\end{aligned}
$$

for all $t>0$ (see Lemma 3.2 (ii)). This together with (2.8) yields

$$
\begin{gathered}
t^{(N / 2)(1-1 / q)}\left\|S(t) \varphi-\sum_{|\alpha| \leq K, \alpha_{N} \notin \mathcal{O}} M_{\alpha}(\varphi, 0) g_{\alpha}(t)\right\|_{q} \\
= \begin{cases}o\left(t^{-K / 2}\right) & \text { if } K=[K], \\
O\left(t^{-K / 2}\right) & \text { if } K>[K],\end{cases}
\end{gathered}
$$

as $t \rightarrow \infty$, for any $q \in[1, \infty]$, which gives higher order asymptotic expansion of $S(t) \varphi$. See also Proposition 3.1.

\subsection{Main results}

In this subsection we give the main theorem, which yields higher order asymptotic expansions of the solutions of (1.1) satisfying (1.3). Furthermore, as 
a corollary, we give the relationship between the decay rate of $\|R(t)\|_{q}$ and the integrability of the initial datum $\varphi$.

Before treating our main theorem, modifying [9], we introduce the functions $U_{n}=U_{n}(x, t)$ defined inductively by

$$
\begin{aligned}
U_{0}(x, t):= & \sum_{|\alpha| \leq K, \alpha_{N} \notin \mathcal{O}}\left(M_{\alpha}(u(t), t)-\int_{0}^{t} N_{\alpha}(\gamma[f(s)], s) d s\right) g_{\alpha}(x, t) \\
& +2 \sum_{|\tilde{\alpha}| \leq K} \int_{0}^{t} G_{1}\left(x_{N}, t-s\right) \tilde{M}_{\tilde{\alpha}}(\gamma[f(s)], s) d s \tilde{g}_{\tilde{\alpha}}\left(x^{\prime}, t\right), \\
U_{n}(x, t):= & U_{0}(x, t)+2 \int_{0}^{t} G_{1}\left(x_{N}, t-s\right)\left(e^{(t-s) \Delta^{\prime}} Q_{K}(s) \gamma\left[F_{n-1}(s)\right]\right) d s,
\end{aligned}
$$

for $n=1,2, \ldots$, where $f(x, t)=\kappa|u(x, t)|^{p-1} u(x, t), F_{n-1}(x, t)=\kappa\left|U_{n-1}(x, t)\right|^{p-1}$. $U_{n-1}(x, t)$, and

$$
\begin{aligned}
N_{\alpha}(\gamma[f(t)], t):= & \int_{\partial \boldsymbol{R}_{+}^{N}} x^{\alpha} \gamma[f(t)]\left(x^{\prime}\right) d \sigma \\
& -\sum_{\rho \in J(\alpha), \rho_{N} \notin \mathcal{O}} N_{\rho}(\gamma[f(t)], t) \int_{\boldsymbol{R}_{+}^{N}} x^{\alpha} g_{\rho}(x, t) d x .
\end{aligned}
$$

Now we are ready to state the main theorem of this paper.

Theorem 2.1. Consider the initial-boundary value problem (1.1) under condition (1.2) for some $K \geq 0$ and $A_{p}>1 / 2$. Let $u$ be a unique solution of (1.1) satisfying (1.3), and let $n=0,1,2, \ldots$ Then there holds the following:

(i) The function $U_{n}$ defined by (2.11) satisfies

$$
\begin{aligned}
& \sup _{t>0}(1+t)^{(N / 2)(1-1 / q)}\left[\left\|U_{n}(t)\right\|_{q}+t^{1 /(2 q)}\left\|U_{n}(t)\right\|_{q, \partial \boldsymbol{R}_{+}^{N}}\right]<\infty, \\
& \sup _{t>0}(1+t)^{-l / 2}\left[\left\|\mid U_{n}(t)\right\|_{l}+t^{1 / 2}\left\|U_{n}(t)\right\| \|_{l, \partial \boldsymbol{R}_{+}^{N}}\right]<\infty,
\end{aligned}
$$

for any $q \in[1, \infty]$ and $l \in[0, K]$;

(ii) For any $q \in[1, \infty]$,

$$
\begin{aligned}
& t^{(N / 2)(1-1 / q)}\left[\left\|u(t)-U_{n}(t)\right\|_{q}+t^{1 /(2 q)}\left\|u(t)-U_{n}(t)\right\|_{q, \partial \boldsymbol{R}_{+}^{N}}\right] \\
& \quad \preceq \begin{cases}(1+t)^{-K / 2}+(1+t)^{-(n+1)\left(A_{p}-1 / 2\right)} & \text { if }(n+1)\left(2 A_{p}-1\right) \neq K, \\
(1+t)^{-K / 2} \log (2+t) & \text { if }(n+1)\left(2 A_{p}-1\right)=K,\end{cases}
\end{aligned}
$$

for all $t>0$; 
(iii) If $(n+1)\left(2 A_{p}-1\right)>K$, then, for any $q \in[1, \infty]$,

$$
\begin{gathered}
t^{(N / 2)(1-1 / q)}\left[\left\|u(t)-U_{n}(t)\right\|_{q}+t^{1 /(2 q)}\left\|u(t)-U_{n}(t)\right\|_{q, \partial \boldsymbol{R}_{+}^{N}}\right] \\
= \begin{cases}o\left(t^{-K / 2}\right) & \text { if } K=[K], \\
O\left(t^{-K / 2}\right) & \text { if } K>[K],\end{cases}
\end{gathered}
$$

as $t \rightarrow \infty$;

(iv) For any $l \in[0, K]$ and $\sigma>0$,

$$
\begin{array}{r}
(1+t)^{-l / 2}\left[\left\|\mid u(t)-U_{n}(t)\right\|_{l}+t^{1 / 2}\left\|u(t)-U_{n}(t)\right\| \|_{l, \partial \boldsymbol{R}_{+}^{N}}\right] \\
\preceq(1+t)^{-K / 2+\sigma}+(1+t)^{-(n+1)\left(A_{p}-1 / 2\right)}, \quad t>0 .
\end{array}
$$

By Theorem 2.1 we see that the functions $U_{0}$ and $\left\{U_{n}\right\}_{n=1}^{\infty}$ give a linear approximation and nonlinear approximations to the solution $u$, respectively.

Remark 2.1. (i) Since, for any multi-index $\alpha \in N_{0}^{N}$, it holds that

$$
\left.\frac{1}{\alpha !} \partial_{y}^{\alpha} \Gamma(x, y, 1+t)\right|_{y=0}= \begin{cases}g_{\alpha}(x, t) & \text { if } \alpha_{N} \notin \mathcal{O}, \\ 0 & \text { if } \alpha_{N} \in \mathcal{O},\end{cases}
$$

the first term of $U_{0}$ is represented as a linear combination of $\left\{\partial_{y}^{\alpha} \Gamma(x, y\right.$, $\left.1+t)\left.\right|_{y=0}\right\}_{|\alpha| \leq K}$, and plays a role of projection of the solution onto the space spanned by $\left\{\left.\partial_{y}^{\alpha} \Gamma(x, y, 1+t)\right|_{y=0}\right\}_{|\alpha| \leq K}$.

(ii) If $(n+1)\left(2 A_{p}-1\right)>K$, then the decay estimate of $\left\|u(t)-U_{n}(t)\right\|_{q}$ as $t \rightarrow \infty$ in $(2.16)$ is the same as in (2.10).

(iii) $U_{n}(n=1,2, \ldots)$ gives the $([K]+2)$-th order asymptotic expansion of the solution $u$ and is determined systematically by the function $U_{0}$.

As a corollary of Theorem 2.1, we can obtain the following result, which gives a precise estimate on the difference between the solution and its first asymptotic profiles. Compare with (1.7) and (1.9).

Corollary 2.1. Assume the same conditions as in Theorem 2.1 and $K>0$. Let $R$ be the function defined by (1.8). Then there holds the following:

(i) If $K \in(0,1]$, then, for any $q \in[1, \infty]$,

$$
\begin{aligned}
& t^{(N / 2)(1-1 / q)}\|R(t)\|_{q} \\
& \quad=\left\{\begin{array}{ll}
O\left(t^{-K / 2}\right)+O\left(t^{-\left(A_{p}-1 / 2\right)}\right) & \text { if } 2 A_{p}-1 \neq K, \\
O\left(t^{-K / 2} \log t\right) & \text { if } 2 A_{p}-1=K,
\end{array} \quad \text { as } t \rightarrow \infty ;\right.
\end{aligned}
$$

(ii) If $K>1$, then, for any $q \in[1, \infty]$,

$$
t^{(N / 2)(1-1 / q)}\|R(t)\|_{q}=O\left(t^{-1 / 2}\right)+O\left(t^{-\left(A_{p}-1 / 2\right)}\right) \quad \text { as } t \rightarrow \infty .
$$


Remark 2.2. Consider the initial-boundary value problem (1.1) with the nonlinear term $\kappa|u|^{p-1} u$ replaced by $F(x, t, u)$ satisfying

$$
|F(x, t, u(x, t))| \leq C(1+t)^{-A}|u(x, t)|, \quad(x, t) \in \overline{\boldsymbol{R}_{+}^{N}} \times(0, \infty),
$$

for some constant $C>0$ and $A>1 / 2$. Then, modifying the argument in Subsection 3.2 (see also [8] and [10]), we can prove that there exists a solution satisfying (1.3). Furthermore if the nonlinear term $F$ satisfies

$$
\left\{\begin{array}{l}
\text { (i) } F(x, t, 0)=0 \text { for all }(x, t) \in \overline{\boldsymbol{R}_{+}^{N}} \times(0, \infty) ; \\
\text { (ii) } \text { For any } v_{1} \text { and } v_{2} \in \mathscr{S}, \text { there exists a constant } C \text { such that } \\
\left|F\left(x, t, v_{1}(x, t)\right)-F\left(x, t, v_{2}(x, t)\right)\right| \leq C(1+t)^{-A}\left|v_{1}(x, t)-v_{2}(x, t)\right| \\
\quad \text { for all }(x, t) \in \overline{\boldsymbol{R}_{+}^{N}} \times(0, \infty),
\end{array}\right.
$$

then there holds the conclusion of Theorem 2.1. Here

$$
\begin{aligned}
& \mathscr{S}:=\left\{v \in C\left(\overline{\boldsymbol{R}_{+}^{N}} \times(0, \infty)\right) \cap L^{\infty}\left(0, \infty: L^{\infty}\left(\boldsymbol{R}_{+}^{N}\right)\right): \sup _{t>0}(1+t)^{(N / 2)(1-1 / q)}\right. \\
&\left.\times\left[\|v(t)\|_{q}+t^{1 /(2 q)}\|v(t)\|_{q, \partial \boldsymbol{R}_{+}^{N}}\right]<\infty \text { for any } q \in[1, \infty]\right\} .
\end{aligned}
$$

\section{Preliminary results}

In this section we recall some properties on the solution of the heat equation and operators $P_{K}(t)$ and $Q_{K}(t)$. Furthermore we give some weighted $L^{1}$ estimates for the solution $u$ of (1.1) satisfying (1.3).

\subsection{Preliminaries}

In this subsection we recall some preliminary results of $S(t) \varphi$ and $e^{t \Delta^{\prime}} \psi$ given in (2.1) and (2.2) respectively. Furthermore we give some preliminary results on the operators $P_{K}(t)$ and $Q_{K}(t)$ defined by (2.4) and (2.6).

For any $\alpha \in N_{0}^{N}$ and $j \in N_{0}$, then there exists a constant $C_{1}$ such that

$$
\left|\partial_{t}^{j} \partial_{x}^{\alpha} G_{N}(x, t)\right| \leq C_{1} t^{-(N+|\alpha|+2 j) / 2}\left[1+\left(\frac{|x|}{t^{1 / 2}}\right)\right]^{|\alpha|+2 j} \exp \left(-\frac{|x|^{2}}{4 t}\right)
$$

for all $(x, t) \in \boldsymbol{R}^{N} \times(0, \infty)$. This inequality together with (1.5) yields the inequalities

$$
\begin{aligned}
& \left\|g_{\alpha}(t)\right\|_{q} \preceq(1+t)^{-(N / 2)(1-1 / q)-|\alpha| / 2}, \\
& \int_{\boldsymbol{R}_{+}^{N}}|x|^{m}\left|g_{\alpha}(x, t)\right| d x \preceq(1+t)^{(m-|\alpha|) / 2}
\end{aligned}
$$




$$
\begin{aligned}
& \left\|g_{\alpha}(t)\right\|_{q, \partial \boldsymbol{R}_{+}^{N}} \preceq(1+t)^{-(N / 2)(1-1 / q)-1 /(2 q)-|\alpha| / 2}, \\
& \int_{\partial \boldsymbol{R}_{+}^{N}}|x|^{m}\left|g_{\alpha}(x, t)\right| d \sigma \preceq(1+t)^{(m-|\alpha|-1) / 2}, \\
& \left\|\tilde{g}_{\tilde{\alpha}}(t)\right\|_{q, \boldsymbol{R}^{N-1}} \preceq(1+t)^{-((N-1) / 2)(1-1 / q)-|\tilde{\alpha}| / 2}, \\
& \int_{\boldsymbol{R}^{N-1}}\left|x^{\prime}\right|^{m} \tilde{g}_{\tilde{\alpha}}\left(x^{\prime}, t\right) \mid d x^{\prime} \preceq(1+t)^{(m-|\tilde{\alpha}|) / 2},
\end{aligned}
$$

for all $t>0$ and any $q \in[1, \infty]$ and $m \geq 0$. Furthermore, by (2.1) and (2.2) we have the following (see also Lemma 2.1 in [6]):

$(S 1)$ There exists a constant $C_{2}$ such that, for any $1 \leq r \leq q \leq \infty$,

$$
\|S(t) \varphi\|_{q} \leq C_{2} t^{-(N / 2)(1 / r-1 / q)}\|\varphi\|_{r}, \quad t>0 .
$$

Furthermore, for any $l \geq 0$ and $\delta>0$, there exists a constant $C_{3}$ such that

$$
\int_{\boldsymbol{R}_{+}^{N}}|x|^{l}|(S(t) \varphi)(x)| d x \leq(1+\delta) \int_{\boldsymbol{R}_{+}^{N}}|x|^{l}|\varphi(x)| d x+C_{3} t^{l / 2} \int_{\boldsymbol{R}_{+}^{N}}|\varphi(x)| d x, \quad t>0 .
$$

This inequality implies that

$$
\|S(t) \varphi\|_{l} \leq(1+\delta)\|\varphi\|_{l}+C_{4}\left(1+t^{l / 2}\right)\|\varphi\|_{1}, \quad t>0,
$$

for some constant $C_{4}$;

(S2) There exists a constant $C_{5}$ such that, for any $q \in[1, \infty]$,

$$
\|S(t) \varphi\|_{q, \partial \boldsymbol{R}_{+}^{N}} \leq C_{5} t^{-(N / 2)(1-1 / q)-1 /(2 q)}\|\varphi\|_{1}, \quad t>0 .
$$

Furthermore, for any $l \geq 0$ and $\delta>0$, there exists a constant $C_{6}$ such that

$$
\begin{aligned}
\int_{\boldsymbol{R}^{N-1}}\left|x^{\prime}\right|^{l}\left|(S(t) \varphi)\left(x^{\prime}, 0\right)\right| d x \leq & (1+\delta) t^{-1 / 2} \int_{\boldsymbol{R}_{+}^{N}}|x|^{l}|\varphi(x)| d x \\
& +C_{6} t^{(l-1) / 2} \int_{\boldsymbol{R}_{+}^{N}}|\varphi(x)| d x, \quad t>0 .
\end{aligned}
$$

This inequality implies that

$$
\|\mid S(t) \varphi\|_{l, \partial \boldsymbol{R}_{+}^{N}} \leq(1+\delta) t^{-1 / 2}\|\| \varphi\left\|_{l}+C_{7} t^{-1 / 2}\left(1+t^{l / 2}\right)\right\| \varphi \|_{1}, \quad t>0,
$$

for some constant $C_{7}$;

(S3) There exists a constant $C_{8}$ such that, for any $1 \leq r \leq q \leq \infty$,

$$
\left\|e^{t \Delta^{\prime}} \psi\right\|_{q, \boldsymbol{R}^{N-1}} \leq C_{8} t^{-((N-1) / 2)(1 / r-1 / q)}\|\psi\|_{r, \boldsymbol{R}^{N-1}}, \quad t>0 .
$$

Furthermore, for any $l \geq 0$ and $\delta>0$, there exists a constant $C_{9}$ such that 


$$
\begin{aligned}
\int_{\boldsymbol{R}^{N-1}}\left|x^{\prime}\right|^{l}\left|\left(e^{t \Delta^{\prime}} \psi\right)\left(x^{\prime}\right)\right| d x^{\prime} \leq & (1+\delta) \int_{\boldsymbol{R}^{N-1}}\left|x^{\prime}\right|^{l}\left|\psi\left(x^{\prime}\right)\right| d x^{\prime} \\
& +C_{9} t^{l / 2} \int_{\boldsymbol{R}^{N-1}}\left|\psi\left(x^{\prime}\right)\right| d x^{\prime}, \quad t>0 .
\end{aligned}
$$

This inequality implies that

$$
\left\|e^{t \Delta^{\prime}} \psi \mid\right\|_{l, \boldsymbol{R}^{N-1}} \leq(1+\delta)\|\| \psi\left\|_{l, \boldsymbol{R}^{N-1}}+C_{10}\left(1+t^{l / 2}\right)\right\| \psi \|_{1, \boldsymbol{R}^{N-1}}, \quad t>0,
$$

for some constant $C_{10}$.

Applying a similar argument as in the proof of [6, Lemmata 2.2 and 2.5] with (2.18), we have the following lemma.

Lemma 3.1. Let $k \geq 0$. Assume $\varphi \in L_{k}^{1}$ and $\psi \in L_{k, \boldsymbol{R}^{N-1}}^{1}$. Then there holds the following:

(i) If it holds that

$$
\int_{\boldsymbol{R}_{+}^{N}} x^{\alpha} \varphi(x) d x=0, \quad|\alpha| \leq k \text { with } \alpha_{N} \notin \mathcal{O},
$$

then, for any $l \in[0, k-[k]]$, there exists a constant $C_{1}$ such that

$$
\int_{\boldsymbol{R}_{+}^{N}}|x|^{l}|(S(t) \varphi)(x)| d x \leq C_{1} t^{-(k-l) / 2} \int_{\boldsymbol{R}_{+}^{N}}|x|^{k}|\varphi(x)| d x, \quad t>0 .
$$

In particular, if $k=[k]$, then $\lim _{t \rightarrow \infty} t^{k / 2}\|S(t) \varphi\|_{1}=0$.

(ii) If it holds that

$$
\int_{\boldsymbol{R}^{N-1}}\left(x^{\prime}\right)^{\tilde{\alpha}} \psi\left(x^{\prime}\right) d x^{\prime}=0, \quad|\tilde{\alpha}| \leq k,
$$

then, for any $l \in[0, k-[k]]$, there exists a constant $C_{3}$ such that

$$
\int_{\boldsymbol{R}^{N-1}}\left|x^{\prime}\right|^{l}\left|\left(e^{t \Delta^{\prime}} \psi\right)\left(x^{\prime}\right)\right| d x^{\prime} \leq C_{3} t^{-(k-l) / 2} \int_{\boldsymbol{R}^{N-1}}\left|x^{\prime}\right|^{k}\left|\psi\left(x^{\prime}\right)\right| d x^{\prime}, \quad t>0 .
$$

In particular, if $k=[k]$, then $\lim _{t \rightarrow \infty} t^{k / 2}\left\|e^{t \Delta^{\prime}} \psi\right\|_{1, \boldsymbol{R}^{N-1}}=0$.

Next we give the following lemma on the operator $P_{k}(t)$ (see also [6, Lemma 2.3]).

Lemma 3.2. Let $k \geq 0$ and $f=f(x, t) \in C\left(\overline{\boldsymbol{R}_{+}^{N}} \times(0, \infty)\right)$ be a bounded function such that $\sup _{0<\tau<t} \tau^{1 / 2}\|f(\tau) \mid\|_{k, \partial \boldsymbol{R}_{+}^{N}}<\infty$ for all $t>0$. Let $\varphi \in X_{k}\left(\boldsymbol{R}_{+}^{N}\right)$ and $u$ be a solution of the initial-boundary value problem

$$
\begin{aligned}
& \partial_{t} u=\Delta u \quad \text { in } \boldsymbol{R}_{+}^{N} \times(0, \infty), \quad \partial_{v} u=f(x, t) \quad \text { on } \partial \boldsymbol{R}_{+}^{N} \times(0, \infty), \\
& u(x, 0)=\varphi(x) \text { in } \boldsymbol{R}_{+}^{N} .
\end{aligned}
$$


Then there holds the following:

(i) The function $v=\left[P_{k}(t) u(t)\right](x)$ satisfies

$$
\begin{cases}\partial_{t} v=\Delta v-\sum_{|\alpha| \leq k, \alpha_{N} \notin \mathcal{O}} N_{\alpha}(\gamma[f(t)], t) g_{\alpha}(x, t) & \text { in } \boldsymbol{R}_{+}^{N} \times(0, \infty), \\ \partial_{v} v=f(x, t) & \text { on } \partial \boldsymbol{R}_{+}^{N} \times(0, \infty), \\ v(x, 0)=\left(P_{k}(0) u(0)\right)(x) & \text { in } \boldsymbol{R}_{+}^{N} ;\end{cases}
$$

(ii) For any $|\alpha| \leq K$ with $\alpha_{N} \notin \mathcal{O}$,

$$
\frac{d}{d t} M_{\alpha}(u(t), t)=N_{\alpha}(\gamma[f(t)], t), \quad t>0
$$

Proof. Following the idea of the proof of Lemma 2.3 in [6], we prove this lemma. We first prove (3.4). For $|\alpha| \leq 1$ with $\alpha_{N}=0$, by (2.5) we have

$$
M_{\alpha}(u(t), t)=\int_{\boldsymbol{R}_{+}^{N}}\left(x^{\prime}\right)^{\alpha} u(x, t) d x .
$$

Since $\nabla\left(x^{\prime}\right)^{\alpha} \cdot v=0$ for $v=(0, \ldots, 0,1)$, we obtain

$$
\begin{aligned}
\frac{d}{d t} M_{\alpha, 2 \lambda}(u(t), t) & =\int_{\boldsymbol{R}_{+}^{N}}\left(x^{\prime}\right)^{\alpha} u_{t}(x, t) d x=\int_{\boldsymbol{R}_{+}^{N}}\left(x^{\prime}\right)^{\alpha} \Delta u(x, t) d x \\
& =\int_{\partial \boldsymbol{R}_{+}^{N}}\left(x^{\prime}\right)^{\alpha} f(x, t) d \sigma-\int_{\boldsymbol{R}_{+}^{N}} \nabla\left(x^{\prime}\right)^{\alpha} \cdot \nabla u(x, t) d x=N_{\alpha}(\gamma[f(t)], t) .
\end{aligned}
$$

So we have (3.4) for the cases $|\alpha| \leq 1$ with $\alpha_{N}=0$.

Let $k \geq 2$ and $n \in\{0, \ldots,[k]-1\}$, and assume

$$
\frac{d}{d t} M_{\alpha}(u(t), t)=N_{\alpha}(\gamma[f(t)], t), \quad|\alpha| \leq n \text { with } \alpha_{N} \notin \mathcal{O} .
$$

We recall that, by (2.4) and (2.8), for any $|\alpha| \leq k$ with $\alpha_{N} \notin \mathcal{O}$ and $\zeta \in X_{k}\left(\boldsymbol{R}_{+}^{N}\right)$, it holds that

$$
\begin{aligned}
\int_{\boldsymbol{R}_{+}^{N}}\left(\Delta x^{\alpha}\right) \zeta(x) d x-\sum_{\rho \in J(\alpha), \rho_{N} \notin \mathcal{O}} M_{\rho}(\zeta, t) \int_{\boldsymbol{R}_{+}^{N}}\left(\Delta x^{\alpha}\right) g_{\rho}(t) d x \\
\quad=\int_{\boldsymbol{R}_{+}^{N}}\left(\Delta x^{\alpha}\right)\left[\zeta(x)-\sum_{|\rho| \leq|\alpha|-1, \rho_{N} \notin \mathcal{O}} M_{\rho}(\zeta, t) g_{\rho}(t)\right] d x=0, \quad t>0 .
\end{aligned}
$$


This together with (2.5), (2.12), and (3.5) implies that, for $|\alpha|=n+1$ with $\alpha_{N} \notin \mathcal{O}$,

$$
\begin{aligned}
\frac{d}{d t} M_{\alpha}(u(t), t)= & \int_{\boldsymbol{R}_{+}^{N}} x^{\alpha} u_{t}(x, t) d x-\frac{d}{d t} \sum_{\rho \in J(\alpha), \rho_{N} \notin \mathcal{O}} M_{\rho}(u(t), t) \int_{\boldsymbol{R}_{+}^{N}} x^{\alpha} g_{\rho}(x, t) d x \\
= & \int_{\partial \boldsymbol{R}_{+}^{N}} x^{\alpha} f(x, t) d \sigma-\sum_{\rho \in J(\alpha), \rho_{N} \notin \mathcal{O}} \frac{d}{d t} M_{\rho}(u(t), t) \int_{\boldsymbol{R}_{+}^{N}} x^{\alpha} g_{\rho}(x, t) d x \\
& +\int_{\boldsymbol{R}_{+}^{N}}\left(\Delta x^{\alpha}\right) u(x, t) d x-2 \sum_{\rho \in J(\alpha), \rho_{N} \notin \mathcal{O}} M_{\rho}(u(t), t) \int_{\boldsymbol{R}_{+}^{N}}\left(\Delta x^{\alpha}\right) g_{\rho}(x, t) d x \\
= & \int_{\partial \boldsymbol{R}_{+}^{N}} x^{\alpha} f(x, t) d \sigma-\sum_{\rho \in J(\alpha), \rho_{N} \notin \mathcal{O}} N_{\rho}(\gamma[f(t)], t) \int_{\boldsymbol{R}_{+}^{N}} x^{\alpha} g_{\rho}(x, t) d x \\
& +\int_{\boldsymbol{R}_{+}^{N}}(\Delta x)^{\alpha}\left(u(x, t)-\sum_{|\rho| \leq n, \rho_{N} \notin \mathcal{O}} M_{\rho}(u(t), t) g_{\rho}(x, t)\right) d x \\
= & N_{\alpha}(\gamma[f(t)], t)
\end{aligned}
$$

for all $t>0$. Therefore, by the inductive argument with respect to $n$ we obtain (3.4) for all $|\alpha| \leq k$ with $\alpha_{N} \notin \mathcal{O}$.

Next we prove (3.3). By (2.4) and (3.4) we have

$$
\partial_{t} v-\Delta v=-\sum_{|\alpha| \leq k, \alpha_{N} \notin \mathcal{O}} \frac{d}{d t} M_{\alpha}(u(t), t) g_{\alpha}(x, t)=-\sum_{|\alpha| \leq k, \alpha_{N} \notin \mathcal{O}} N_{\alpha}(\gamma[f(t)], t) g_{\alpha}(x, t)
$$

for all $(x, t) \in \boldsymbol{R}_{+}^{N} \times(0, \infty)$. Furthermore we put $h(x)=e^{-c x^{2}}$ for some positive constant $c$. Then, for any $n \in N$, we have

$$
h^{(n+1)}(x)=-2 c n h^{(n-1)}(x)-2 c x h^{(n)}(x), \quad x \in \boldsymbol{R} .
$$

Therefore, since $\partial_{x_{N}} g_{\alpha}(x, t)=0$ on $\partial \boldsymbol{R}_{+}^{N} \times(0, \infty)$ for any multi-index $\alpha \in \boldsymbol{N}_{0}^{N}$ with $\alpha_{N}=0$, we obtain

$$
\partial_{v} v=f(x, t)-\sum_{|\alpha| \leq k, \alpha_{N} \notin \mathcal{O}} M_{\alpha}(u(t), t)\left(-\partial_{x_{N}}\right) g_{\alpha}(x, t)=f(x, t)
$$

for all $(x, t) \in \partial \boldsymbol{R}_{+}^{N} \times(0, \infty)$. Thus we have (3.3), and the proof of Lemma 3.2 is complete.

By Lemmata 3.1 and 3.2 we obtain the asymptotic expansions of the solutions of the heat equation on $\boldsymbol{R}_{+}^{N}$ with the homogeneous Neumann boundary condition. 
Proposition 3.1. Let $\varphi \in X_{K}\left(\boldsymbol{R}_{+}^{N}\right)$ for some $K \geq 0$ and $u(x, t)=(S(t) \varphi)(x)$. Put

$$
U_{\text {lin }}(x, t)=\sum_{|\alpha| \leq K, \alpha_{N} \notin \mathcal{O}} M_{\alpha}(\varphi, 0) g_{\alpha,}(x, t) .
$$

Then, for any $q \in[1, \infty]$,

$$
\begin{aligned}
& t^{(N / 2)(1-1 / q)}\left(\left\|u(t)-U_{\text {lin }}(t)\right\|_{q}+t^{1 /(2 q)}\left\|u(t)-U_{\text {lin }}(t)\right\|_{q, \partial \boldsymbol{R}_{+}^{N}}\right) \\
& = \begin{cases}o\left(t^{-K / 2}\right) & \text { if } K=[K], \\
O\left(t^{-K / 2}\right) & \text { if } K>[K],\end{cases}
\end{aligned}
$$

as $t \rightarrow \infty$.

Proof. Put

$$
v(x, t):=P_{K}(t) u(t)=u(x, t)-\sum_{|\alpha| \leq K, \alpha_{N} \neq \mathcal{O}} M_{\alpha}(u(t), t) g_{\alpha}(x, t) .
$$

Then, by Lemma 3.2 (i) and (2.8) we see that the function $v$ is a solution of the heat equation in $\boldsymbol{R}_{+}^{N}$ with the homogeneous Neumann boundary condition such that

$$
\int_{\boldsymbol{R}_{+}^{N}} x^{\alpha} v(x, t) d x=0, \quad|\alpha| \leq K \text { with } \alpha_{x_{N}} \notin \mathcal{O},
$$

for all $t \geq 0$. This together with Lemma 3.1 implies

$$
\|v(t)\|_{1}=o\left(t^{-K / 2}\right) \quad \text { if } K=[K], \quad\|v(t)\|_{1}=O\left(t^{-K / 2}\right) \quad \text { if } K>[K],
$$

as $t \rightarrow \infty$. On the other hand, by Lemma 3.2 (ii) with $f \equiv 0$ we have

$$
v(x, t)=u(x, t)-U_{\text {lin }}(x, t) \quad \text { in } \overline{\boldsymbol{R}_{+}^{N}} \times(0, \infty) .
$$

Therefore, since $v(2 t)=S(t) v(t)$, Proposition 3.1 follows from $(S 1),(S 2)$, (3.6), and (3.7).

We put

$$
D[\psi](x, t)=2 G_{1}\left(x_{N}, t\right)\left(e^{t \Delta^{\prime}} \psi\right)\left(x^{\prime}\right), \quad x \in \boldsymbol{R}_{+}^{N}, t>0 .
$$

Then, for any $q \in[1, \infty]$,

$$
\|D[\psi](t)\|_{q}+t^{1 /(2 q)}\|D[\psi](t)\|_{q, \partial \boldsymbol{R}_{+}^{N}} \preceq t^{-(N / 2)(1-1 / q)}\|\psi\|_{1, \boldsymbol{R}^{N-1}}, \quad t>0 .
$$

Furthermore, by $(S 3)$, for any $l \in[0, k]$, we have 


$$
\begin{aligned}
\int_{\boldsymbol{R}_{+}^{N}}|x|^{l}|D[\psi](x, t)| d x & \preceq \int_{\boldsymbol{R}^{N-1}}\left(\int_{0}^{\infty}\left(\left|x^{\prime}\right|^{l}+x_{N}^{l}\right) G_{1}\left(x_{N}, t\right) d x_{N}\right)\left(e^{t \Delta^{\prime}} \psi\right)\left(x^{\prime}\right) d x^{\prime} \\
& \preceq \int_{\partial \boldsymbol{R}^{N-1}}\left|x^{\prime}\right|^{l}\left|\psi\left(x^{\prime}\right)\right| d x^{\prime}+t^{l / 2}\|\psi\|_{1, \boldsymbol{R}^{N-1}}, \quad t>0 .
\end{aligned}
$$

Similarly we obtain

$$
\begin{aligned}
\int_{\partial \boldsymbol{R}_{+}^{N}}|x|^{l}|D[\psi](x, t)| d \sigma \preceq & t^{-1 / 2} \int_{\partial \boldsymbol{R}^{N-1}}\left|x^{\prime}\right|^{l}\left|\psi\left(x^{\prime}\right)\right| d x^{\prime} \\
& +t^{(l-1) / 2}\|\psi\|_{1, \boldsymbol{R}^{N-1}}, \quad t>0 .
\end{aligned}
$$

These inequalities yield

$$
\begin{aligned}
& \|\| D[\psi](t)\|\|_{l}+t^{1 / 2}\|D[\psi](t)\| \|_{l, \partial \boldsymbol{R}_{+}^{N}} \\
& \quad \preceq\|\| \psi \mid\left\|_{l, \boldsymbol{R}^{N-1}}+\left(1+t^{l / 2}\right)\right\| \psi \|_{1, \boldsymbol{R}^{N-1}, \quad}, \quad t>0 .
\end{aligned}
$$

At the end of this section, we give the following lemma on the operator $Q_{k}(t)$ (see also [9, Lemma 2.3]).

Lemma 3.3. Let $k \geq 0$ and $f=f(x, t) \in C\left(\overline{\boldsymbol{R}_{+}^{N}} \times(0, \infty)\right)$ be a bounded function such that $\sup _{0<\tau<t} \tau^{1 / 2}\left|\|f(\tau) \mid\|_{k, \partial \boldsymbol{R}_{+}^{N}}<\infty\right.$ for all $t>0$. Then there holds the following:

(i) Assume that there exists a constant $\mu \geq 0$ such that

$$
\sup _{t>0}(1+t)^{-l / 2+\mu} t^{1 / 2}\|f f(t)\|_{l, \partial \boldsymbol{R}_{+}^{N}}<\infty
$$

for all $l \in[0, k]$. Then, for any $|\tilde{\alpha}| \leq k$, there exists a constant $C_{1}$ such that

$$
\left|\tilde{M}_{\tilde{\alpha}}(\gamma[f(t)], t)\right| \leq C_{1}(1+t)^{|\tilde{\alpha}| / 2-\mu} t^{-1 / 2}, \quad t>0 .
$$

Furthermore

$$
\sup _{t>0}(1+t)^{-l / 2+\mu} t^{1 / 2}\left\|Q_{K}(t) \gamma[f(t)]\right\| \|_{l, \boldsymbol{R}^{N-1}}<\infty
$$

for any $l \in[0, K]$ and $q \in[1, \infty]$;

(ii) Put

$$
\mathscr{D}(x, t)=\int_{0}^{t} D\left[Q_{K}(s)[\gamma[f(s)]](t-s) d s, \quad x \in \boldsymbol{R}_{+}^{N}, t>0,\right.
$$

where $D[\cdot]$ is the function given in (3.8). If there exists a constant $\mu^{\prime} \geq 0$ such that

$$
\sup _{t>0}\left[t^{(N / 2)(1-1 / q)+\mu^{\prime}+1 /(2 q)}\|f(t)\|_{q, \partial \boldsymbol{R}_{+}^{N}}+(1+t)^{-l / 2+\mu^{\prime}} t^{1 / 2}\|f(t) \mid\|_{l, \partial \boldsymbol{R}_{+}^{N}}\right]<\infty
$$


for all $l \in[0, K]$ and $q \in[1, \infty]$, then

$$
\begin{aligned}
& t^{(N / 2)(1-1 / q)}\left(\|\mathscr{D}(t)\|_{q}+t^{1 /(2 q)}\|\mathscr{D}(t)\|_{q, \partial \boldsymbol{R}_{+}^{N}}\right) \\
& \qquad t^{-K / 2} \int_{0}^{t}(1+s)^{K / 2-\mu^{\prime}} s^{-1 / 2} d s, \quad t>0 .
\end{aligned}
$$

Proof. We first prove assertion (i). By (2.7) we can obtain (3.14) for the case $l \in\{0,1\}$ directly. Furthermore, by (2.5) and (3.2) we can prove (3.14) inductively. On the other hand, by (2.6), (3.2), (3.13), and (3.14) we have

$$
\begin{aligned}
\| Q_{K}(t)\left[\gamma[f(t)]\|\|_{l, \boldsymbol{R}^{N-1}}\right. & \leq\left\|f ( t ) \left|\left\|_{l, \partial \boldsymbol{R}_{+}^{N}}+\sum_{|\tilde{\alpha}| \leq K}\left|\tilde{M}_{\tilde{\alpha}}(\gamma[f(t)], t)\right|\right\| \tilde{g}_{\tilde{\alpha}}(t) \|_{l, \boldsymbol{R}^{N-1}}\right.\right. \\
& \preceq\|f(t) \mid\|_{l, \partial \boldsymbol{R}_{+}^{N}}+\sum_{|\tilde{\alpha}| \leq K}(1+t)^{|\tilde{\alpha}| / 2-\mu} t^{-1 / 2}(1+t)^{(l-|\tilde{\alpha}|) / 2} \\
& \preceq(1+t)^{l / 2-\mu} t^{-1 / 2}
\end{aligned}
$$

for all $t>0$. This implies (3.15), and we have assertion (i).

Next we prove assertion (ii). By (3.16) we put

$$
\mathscr{D}(x, t)=\left(\int_{0}^{t / 2}+\int_{t / 2}^{t}\right) D\left[Q_{K}(s)[\gamma[f(s)]](t-s) d s=: I_{1}(x, t)+I_{2}(x, t) .\right.
$$

By (2.9) we can apply Lemma 3.1 (ii), and by (3.9), (3.15) with $\mu=\mu^{\prime}$, and (3.17), for any $q \in[1, \infty]$, we have

$$
\begin{aligned}
t^{(N / 2)(1-1 / q)}\left\|I_{1}(t)\right\|_{q} & \preceq \int_{0}^{t / 2} \| e^{((t-s) / 2) \Delta^{\prime}}\left[Q_{K}(s)[\gamma[f(s)]] \|_{1, \boldsymbol{R}^{N-1}} d s\right. \\
& \preceq \int_{0}^{t / 2}(t-s)^{-K / 2} \| Q_{K}(s)\left[\gamma[f(s)]\|\|_{K, \boldsymbol{R}^{N-1}} d s\right. \\
& \preceq t^{-K / 2} \int_{0}^{t}(1+s)^{K / 2-\mu^{\prime}} s^{-1 / 2} d s, \quad t>0 .
\end{aligned}
$$

Similarly, applying the above argument, for any $q \in[1, \infty]$, it holds that

$$
t^{(N / 2)(1-1 / q)+1 /(2 q)}\left\|I_{1}(t)\right\|_{q, \partial \boldsymbol{R}_{+}^{N}} \preceq t^{-K / 2} \int_{0}^{t}(1+s)^{K / 2-\gamma^{\prime}} s^{-1 / 2} d s, \quad t>0 .
$$

On the other hand, by (3.9) and (3.15) with $(l, \mu)=\left(0, \mu^{\prime}\right)$ we have

$$
\begin{aligned}
\left\|I_{2}(t)\right\|_{1} & \preceq \int_{t / 2}^{t} \| Q_{K}(s)\left[\gamma[f(s)] \|_{1, \boldsymbol{R}^{N-1}} d s\right. \\
& \preceq \int_{t / 2}^{t}(1+s)^{-\mu^{\prime}} s^{-1 / 2} d s \preceq t^{-K / 2} \int_{0}^{t}(1+s)^{K / 2-\mu^{\prime}} s^{-1 / 2} d s, \quad t>0 .
\end{aligned}
$$


Similarly we obtain

$$
t^{1 / 2}\left\|I_{2}(t)\right\|_{1, \partial \boldsymbol{R}_{+}^{N}} \preceq t^{-K / 2} \int_{0}^{t}(1+s)^{K / 2-\gamma^{\prime}} s^{-1 / 2} d s, \quad t>0 .
$$

Furthermore, by (2.6), (3.2), (3.14) with $\mu=\mu^{\prime}$, and (3.17) we see that

$$
\begin{aligned}
\left\|Q_{K}(s)[\gamma[f(s)]]\right\|_{\infty, \boldsymbol{R}^{N-1}} & \leq\|f(s)\|_{\infty, \partial \boldsymbol{R}_{+}^{N}}+\sum_{|\tilde{\alpha}| \leq K}\left|\tilde{M}_{\tilde{\alpha}}(\gamma[f(s)], s)\right|\left\|\tilde{g}_{\tilde{\alpha}}(s)\right\|_{\infty, \boldsymbol{R}^{N-1}} \\
& \preceq s^{-N / 2-\mu^{\prime}}+(1+s)^{-(N-1) / 2-\mu^{\prime}} s^{-1 / 2} \preceq s^{-N / 2-\mu^{\prime}}
\end{aligned}
$$

for all $s>0$. Therefore, by (3.8) we have

$$
\begin{aligned}
t^{N / 2}\left|I_{2}(x, t)\right| & \preceq t^{N / 2} \int_{t / 2}^{t}(t-s)^{-1 / 2}\left\|Q_{K}(s)[\gamma[f(s)]]\right\|_{\infty, \boldsymbol{R}^{N-1}} d s \\
& \preceq t^{1 / 2-\mu^{\prime}} \preceq t^{-K / 2} \int_{0}^{t}(1+s)^{K / 2-\mu^{\prime}} s^{-1 / 2} d s
\end{aligned}
$$

for all $(x, t) \in \overline{\boldsymbol{R}_{+}^{N}} \times(0, \infty)$. This implies that

$$
t^{N / 2}\left(\left\|I_{2}(t)\right\|_{\infty}+\left\|I_{2}(t)\right\|_{\infty, \partial \boldsymbol{R}_{+}^{N}} \preceq t^{-K / 2} \int_{0}^{t}(1+s)^{K / 2-\mu^{\prime}} s^{-1 / 2} d s, \quad t>0 .\right.
$$

Combing (3.22)-(3.24), for any $q \in[1, \infty]$, we have $t^{(N / 2)(1-1 / q)}\left(\left\|I_{2}(t)\right\|_{q}+t^{1 /(2 q)}\left\|I_{2}(t)\right\|_{q, \partial \boldsymbol{R}_{+}^{N}} \preceq t^{-K / 2} \int_{0}^{t}(1+s)^{K / 2-\mu^{\prime}} s^{-1 / 2} d s, \quad t>0\right.$.

This together with (3.19)-(3.21) yields (3.18); thus Lemma 3.3 follows.

\subsection{Weighted $L^{1}$ estimate for the solution of (1.1)}

In this subsection we consider the initial-boundary value problem (1.1) with (1.2) and $A_{p}>1 / 2$. Applying a similar argument as in [8, Section 3], we give some weighted $L^{1}$ estimates of the solution $u$ satisfying (1.3). By using approximate solutions of (1.1) we prove the following lemma, which is a local boundedness of the solution $u$ in the weighted $L^{1}$ space.

Lemma 3.4. Assume condition (1.2) with some $K \geq 0$ and $A_{p}>1 / 2$. Let $u$ be a unique solution of (1.1) satisfying (1.3). Then, for any $T>0$ and $l \in[0, K]$,

$$
\sup _{0<t \leq T}\left(\|u(t)\|_{l}+t^{1 / 2}\|\mid u(t)\|_{l, \partial \boldsymbol{R}_{+}^{N}}\right)<\infty .
$$


Proof. Let $u$ be a unique solution of (1.1) satisfying (1.3). Put

$$
u_{1}(x, t):=(S(t) \varphi)(x), \quad u_{n+1}(x, t):=u_{1}(x, t)+\int_{0}^{t} D\left[\gamma\left[f_{n}(s)\right]\right](x, t-s) d s,
$$

for $(x, t) \in \boldsymbol{R}_{+}^{N} \times(0, \infty)$, where $n=1,2, \ldots$ Here $D[\cdot]$ is the function given in (3.8) and $f_{n}(x, t)=\kappa|u(x, t)|^{p-1} u_{n}(x, t)$. Then, applying the standard parabolic theorem (see, for example, [7, Lemma 2.6]), we see that $u_{n} \in C\left(\overline{\boldsymbol{R}_{+}^{N}} \times(0, \infty)\right) \cap$ $L^{\infty}\left(0, \infty: L^{\infty}\left(\boldsymbol{R}_{+}^{N}\right)\right)$ for any $n=1,2, \ldots$, and

$$
\lim _{n \rightarrow \infty} u_{n}(x, t)=u(x, t)
$$

for all $(x, t) \in \boldsymbol{R}_{+}^{N} \times(0, \infty)$. Let $l \in[0, K]$, and put

$$
w_{n}(t)=\left\|u_{n}(t)\right\|_{l}+t^{1 / 2}\left\|u_{n}(t)\right\|_{l, \partial \boldsymbol{R}_{+}^{N}} .
$$

Then, by $(S 1),(S 2)$, and (3.26) we have

$$
\sup _{0<t<1} w_{1}(t) \leq C_{1}\|\varphi\|_{l}
$$

for some constant $C_{1}$. Furthermore, by (3.26) we obtain

$$
\begin{aligned}
w_{2}(t) \leq & \int_{\boldsymbol{R}_{+}^{N}}(1+|x|)^{l}|(S(t) \varphi)(x)| d x+t^{1 / 2} \int_{\partial \boldsymbol{R}_{+}^{N}}(1+|x|)^{l}|(S(t) \varphi)(x)| d \sigma \\
& +\int_{0}^{t} \int_{\boldsymbol{R}_{+}^{N}}(1+|x|)^{l} D\left[\gamma\left[f_{n}(s)\right]\right](x, t-s) d x d s \\
& +t^{1 / 2} \int_{0}^{t} \int_{\partial \boldsymbol{R}_{+}^{N}}(1+|x|)^{l} D\left[\gamma\left[f_{n}(s)\right]\right](x, t-s) d \sigma d s \\
= & : I_{1}(t)+I_{2}(t)+I_{3}(t), \quad t>0 .
\end{aligned}
$$

Let $T_{1}$ be a sufficiently small constant to be chosen later such that $0<T_{1}<1$. Then, since $I_{1}(t)=w_{1}(t)$, by (3.28) we have

$$
\sup _{0<t \leq T_{1}} I_{1}(t) \leq C_{1}\|\varphi\|_{l} .
$$

On the other hand, by (1.3), (3.12), and (3.28) we obtain

$$
\begin{aligned}
I_{2}(t) & \leq C_{2} \int_{0}^{t}(1+s)^{-A_{p}}\left(1+(t-s)^{l / 2}\right)\left\|u_{1}(s)\right\| \|_{l, \partial \boldsymbol{R}_{+}^{N}} d s \\
& \leq C_{3} \int_{0}^{t} s^{-1 / 2} w_{1}(s) d s \leq C_{1} C_{4} T_{1}^{1 / 2}\|\varphi\|_{l}
\end{aligned}
$$

for all $0<t \leq T_{1}<1$, where $C_{2}, C_{3}$, and $C_{4}$ are constants. Applying a similar argument as above, we see that there exist constants $C_{5}, C_{6}$, and $C_{7}$ such that 


$$
\begin{gathered}
I_{3}(t) \leq C_{5} T_{1}^{1 / 2} \int_{0}^{t}(1+s)^{-A_{p}}(t-s)^{-1 / 2}\left(1+(t-s)^{l / 2}\right)\left\|u_{1}(s)\right\| \|_{l, \partial \boldsymbol{R}_{+}^{N}} d s \\
\leq C_{6} \int_{0}^{t}(t-s)^{-1 / 2} s^{-1 / 2} w_{1}(s) d s \leq C_{1} C_{7} T_{1}^{1 / 2}\|\varphi\|_{l}
\end{gathered}
$$

for all $0<t \leq T_{1}<1$. Combining (3.29)-(3.32) and taking a sufficiently small $T_{1}>0$ so that $\left(C_{4}+C_{7}\right) T_{1}^{1 / 2} \leq 2^{-1}$, we have

$$
\sup _{0<t \leq T_{1}} w_{2}(t) \leq C_{1}\left[1+\left(C_{4}+C_{7}\right) T_{1}^{1 / 2}\right]\|\varphi\|_{l} \leq C_{1}\left(1+2^{-1}\right)\|\varphi\|_{l} .
$$

Repeating the above argument, we obtain

$$
\begin{aligned}
\sup _{0<t \leq T_{1}} w_{n}(t) & \leq C_{1}\left(1+2^{-1}+\cdots+2^{-(n-1)}\right)\|\varphi\|_{l} \\
& \leq 2 C_{1}\|\varphi\|_{l}, \quad n=1,2, \ldots
\end{aligned}
$$

Furthermore, since

$$
\begin{aligned}
& u_{1}(x, t)=\left[S(t-T) u_{1}(T)\right](x), \\
& u_{n+1}(x, t)=\left[S(t-T) u_{n+1}(T)\right](x)+\int_{T}^{t} D\left[\gamma\left[f_{n}(s)\right]\right](x, t-s) d s,
\end{aligned}
$$

for all $(x, t) \in \boldsymbol{R}_{+}^{N} \times(T, \infty)$ and all $T>0$, applying the same argument to (3.34) with $T=T_{1} / 2$, by (3.33) we have

$$
\begin{aligned}
& \sup _{T_{1} / 2<t \leq 3 T_{1} / 2}\left[\left\|u_{n}(t) \mid\right\|_{l}+\left(t-T_{1} / 2\right)^{1 / 2}\left\|u_{n}(t)\right\| \|_{l, \partial \boldsymbol{R}_{+}^{N}}\right] \\
& \quad \leq 2 C_{1}\left\|u_{n}\left(T_{1} / 2\right)\right\|\left\|_{l} \leq\left(2 C_{1}\right)^{2}\right\| \varphi \|_{l}
\end{aligned}
$$

for all $n=1,2, \ldots$. This relation together with (3.33) implies

$\sup _{0<t \leq 3 T_{1} / 2} w_{n}(t) \leq \sup _{0<t \leq T_{1}} w_{n}(t)+\sup _{T_{1}<t \leq 3 T_{1} / 2} w_{n}(t) \leq C_{8}\|\varphi\|_{l}<\infty, \quad n=1,2, \ldots$,

for some constant $C_{8}$. Repeating this argument, for any $T>0$, we obtain

$$
\sup _{n \geq 1} \sup _{0<t \leq T} w_{n}(t)<\infty \text {. }
$$

This together with (3.27) yields (3.25); thus Lemma 3.4 follows.

By Lemma 3.4 we give the main lemma of this section, which is weighted $L^{1}$ estimates of the solution $u$.

Lemma 3.5. Assume the same conditions as in Lemma 3.4. Then, for any $l \in[0, K]$,

$$
\sup _{0<t<\infty}(1+t)^{-l / 2}\left(\|\| u(t) \mid\left\|_{l}+t^{1 / 2}\right\|\|u(t)\|_{l, \partial \boldsymbol{R}_{+}^{N}}\right)<\infty .
$$


Proof. For any $l \in[0, K]$, we put

$$
\mathscr{U}_{l}(t):=\int_{\boldsymbol{R}_{+}^{N}}|x|^{l}|u(x, t)| d x+t^{1 / 2} \int_{\partial \boldsymbol{R}_{+}^{N}}|x|^{l}|u(x, t)| d \sigma .
$$

Let $T$ be a sufficiently large constant to be chosen later such that $T>1$. We put $f(x, t)=\kappa|u(x, t)|^{p-1} u(x, t)$. Since

$$
u(x, t)=[S(t-T) u(T)](x)+\int_{T}^{t} D[\gamma[f(s)]](x, t-s) d s
$$

for all $(x, t) \in \boldsymbol{R}_{+}^{N} \times(T, \infty)$, we have

$$
\begin{aligned}
\mathscr{U}_{l}(t) \leq & \int_{\boldsymbol{R}_{+}^{N}}|x|^{l}|[S(t-T) u(T)](x)| d x \\
& +t^{1 / 2} \int_{\partial \boldsymbol{R}_{+}^{N}}|x|^{l}|[S(t-T) u(T)](x)| d \sigma \\
& +\int_{T}^{t} \int_{\boldsymbol{R}_{+}^{N}}|x|^{l} D[\gamma[f(s)]](x, t-s) d x d s \\
& +t^{1 / 2} \int_{T}^{t} \int_{\partial \boldsymbol{R}_{+}^{N}}|x|^{l} D[\gamma[f(s)]](x, t-s) d \sigma d s \\
= & I_{1}(t)+I_{2}(t)+I_{3}(t)
\end{aligned}
$$

for all $t>T$. By (1.3), (S1), (S2), and (3.25) we obtain

$$
\begin{aligned}
I_{1}(t) \preceq & \left(\int_{\boldsymbol{R}_{+}^{N}}|x|^{l}|u(x, T)| d x+(t-T)^{l / 2}\|u(T)\|_{1}\right) \\
& +t^{1 / 2}\left((t-T)^{-1 / 2} \int_{\boldsymbol{R}_{+}^{N}}|x|^{l}|u(x, T)| d x+(t-T)^{(l-1) / 2}\|u(T)\|_{1}\right) \\
& \preceq t^{l / 2}
\end{aligned}
$$

for all $t>2 T$. Furthermore, it follows from (1.3) and (3.10) that

$$
\begin{aligned}
I_{2}(t) & \preceq \int_{T}^{t} s^{-A_{p}}\left(\int_{\partial \boldsymbol{R}_{+}^{N}}|x|^{l}|u(x, s)| d \sigma+(t-s)^{l / 2}\|u(s)\|_{1, \partial \boldsymbol{R}_{+}^{N}}\right) d s \\
& \preceq \int_{T}^{t} s^{-A_{p}}\left(s^{-1 / 2} \mathscr{U}_{l}(s)+(t-s)^{l / 2} s^{-1 / 2}\right) d s \\
& \preceq T^{-A_{p}+1 / 2} t^{l / 2}\left(\sup _{T<s<t} s^{-l / 2} \mathscr{U}_{l}(s)\right)+t^{l / 2}
\end{aligned}
$$


for all $t>2 T$. Applying the similar argument as above with (3.11), we have

$$
I_{3}(t) \preceq T^{-A_{p}+1 / 2} t^{l / 2}\left(\sup _{T<s<t} s^{-l / 2} \mathscr{U}_{l}(s)\right)+t^{l / 2}
$$

for all $t>2 T$. This together with (3.36)-(3.38) implies that there exists a constant $C$ such that

$$
\sup _{2 T<s<t} s^{-l / 2} \mathscr{U}_{l}(s) \leq C T^{-A_{p}+1 / 2} \sup _{T<s<t} s^{-l / 2} \mathscr{U}_{l}(s)+C
$$

for all $t>2 T \geq 2$. Then, since $A_{p}>1 / 2$, we can take a sufficiently large $T$ so that $C T^{-A_{p}+1 / 2} \leq 1 / 2$ if necessary, and we have

$$
\sup _{2 T<s<\infty} s^{-l / 2} \mathscr{U}_{l}(s) \leq 2 \sup _{T<s<t} s^{-l / 2} \mathscr{U}_{l}(s)+2 C .
$$

This together with (3.25) implies (3.35), and the proof of Lemma 3.5 is complete.

\section{Proof of main results}

In this section we prove Theorem 2.1 and Corollary 2.1. Let $u$ be a unique solution of the initial-boundary value problem (1.1) under condition (1.2) for some $K \geq 0$ and $A_{p}>1 / 2$. Assume (1.3). Then, by Lemma 3.5 we see that the solution $u$ satisfies (3.35). Therefore, for any $|\alpha| \leq K$ with $\alpha_{N} \notin \mathcal{O}$, we can define $M_{\alpha}(u(t), t)$ for all $t \geq 0$, and it holds that

$$
\left|M_{\alpha}(u(t), t)\right| \preceq(1+t)^{|\alpha| / 2}, \quad t \geq 0
$$

(see (2.5)). Furthermore we put

$$
F(x, t):=\kappa|u(x, t)|^{p-1} u(x, t)
$$

for simplicity. Then, by (1.3) and (3.35) we have

$$
\begin{aligned}
& \|F(t)\|_{q, \partial \boldsymbol{R}_{+}^{N}} \preceq(1+t)^{-A_{p}}\|u(t)\|_{q, \partial \boldsymbol{R}_{+}^{N}} \preceq(1+t)^{-A_{p}-(N / 2)(1-1 / q)} t^{-1 /(2 q)}, \\
& \|F(t)\|_{l, \partial \boldsymbol{R}_{+}^{N}} \preceq(1+t)^{-A_{p}}\|u(t)\| \|_{l, \partial \boldsymbol{R}_{+}^{N}} \preceq(1+t)^{-A_{p}+l / 2} t^{-1 / 2},
\end{aligned}
$$

for all $t>0$, where $q \in[1, \infty]$ and $l \in[0, K]$. This together with Lemma 3.3 (i) implies that

$$
\tilde{M}_{\tilde{\alpha}}(\gamma[F(t)], t) \preceq(1+t)^{-A_{p}+|\tilde{\alpha}| / 2} t^{-1 / 2}, \quad t>0,
$$

for any $|\tilde{\alpha}| \leq K$. Furthermore, by (2.12), (3.2), and (4.2) we have

$$
N_{\alpha}(\gamma[F(t)], t) \preceq(1+t)^{-A_{p}+|\alpha| / 2} t^{-1 / 2}, \quad t>0,
$$


for any $|\alpha| \leq K$ with $\alpha_{N} \notin \mathcal{O}$. Therefore, applying Lemma 3.2 (ii), it holds that

$$
\left|M_{\alpha}(u(t), t)-M_{\alpha}\left(u\left(t_{0}\right), t_{0}\right)\right|=\left|\int_{t_{0}}^{t} N_{\alpha}(\gamma[F(s)], s) d s\right| \preceq \int_{t_{0}}^{t}(1+s)^{-A_{p}+|\alpha| / 2} s^{-1 / 2} d s
$$

for all $t \geq t_{0} \geq 0$. This implies the following:

(i) For any $|\alpha| \leq K$ with $\alpha_{N} \notin \mathcal{O}$, if $2 A_{p}>|\alpha|+1$, there exists a constant $M_{\alpha}$ such that

$$
\left|M_{\alpha}(u(t), t)-M_{\alpha}\right| \preceq(1+t)^{-A_{p}+(|\alpha|+1) / 2} \quad \text { for all } t>0 ;
$$

(ii) For any $|\alpha| \leq K$ with $\alpha_{N} \notin \mathcal{O}$, if $1<2 A_{p} \leq|\alpha|+1$, then

$$
M_{\alpha}(u(t), t)=\left\{\begin{array}{ll}
O\left(t^{-A_{p}+(|\alpha|+1) / 2}\right) & \text { if } 2 A_{p}<|\alpha|+1, \\
O(\log t) & \text { if } 2 A_{p}=|\alpha|+1,
\end{array} \quad \text { as } t \rightarrow \infty .\right.
$$

Now we are ready to prove our main theorem. We first prove assertions (i), (ii), and (iv) of Theorem 2.1.

Proof of assertions (i), (ii), and (iv) of Theorem 2.1. Substituting (4.1), (4.3), and (4.4) to the function $U_{0}$ (see (2.11)) we have

$$
\begin{aligned}
\left|U_{0}(x, t)\right| \leq & \sum_{|\alpha| \leq K, \alpha_{N} \notin \mathcal{O}}\left(\left|M_{\alpha}(u(t), t)\right|+\int_{0}^{t}\left|N_{\alpha}(\gamma[f(s)], s)\right| d s\right)\left|g_{\alpha}(x, t)\right| \\
& +2 \sum_{|\tilde{\alpha}| \leq K} \int_{0}^{t} G_{1}\left(x_{N}, t-s\right)\left|\tilde{M}_{\tilde{\alpha}}(\gamma[f(s)], s)\right| d s\left|\tilde{g}_{\tilde{\alpha}}\left(x^{\prime}, t\right)\right| \\
\preceq & \sum_{|\alpha| \leq K, \alpha_{N} \notin \mathcal{O}}\left((1+t)^{|\alpha| / 2}+\int_{0}^{t}(1+s)^{-A_{p}+|\alpha| / 2} s^{-1 / 2}\right)\left|g_{\alpha}(x, t)\right| \\
& +\sum_{|\tilde{\alpha}| \leq K} \int_{0}^{t}(1+s)^{-A_{p}+|\tilde{\alpha}| / 2} s^{-1 / 2} G_{1}\left(x_{N}, t-s\right) d s\left|\tilde{g}_{\tilde{\alpha}}\left(x^{\prime}, t\right)\right|
\end{aligned}
$$

for all $(x, t) \in \overline{\boldsymbol{R}_{+}^{N}} \times(0, \infty)$. Since $A_{p}>1 / 2$ and

$$
\int_{0}^{t}(t-s)^{-1 / 2}(1+s)^{-A_{p}} s^{-1 / 2} d s \preceq t^{-1 / 2}, \quad t>0,
$$

combining (3.2) and (4.7) we obtain (2.13) and (2.14) for the case $n=0$, and assertion (i) follows for the case $n=0$.

Let $n=-1,0,1,2, \ldots$ We assume, without loss of generality, that $\sigma \in$ $\left(0, A_{p}-1 / 2\right)$. Put

$$
\sigma_{n}=\left\{\begin{array}{ll}
\sigma & \text { if } n\left(2 A_{p}-1\right) \geq K, \\
(K / 2)-n\left(A_{p}-1 / 2\right) & \text { if } n\left(2 A_{p}-1\right)<K,
\end{array} \quad \mu_{n}=A_{p}+\frac{K}{2}-\sigma_{n} .\right.
$$


Let $U_{-1} \equiv 0$ in $\overline{\boldsymbol{R}_{+}^{N}} \times(0, \infty)$. Then $(2.11)$ holds for $n=0,1,2, \ldots$ Furthermore, since the solution $u$ satisfies (1.3) and (3.35), assertions (i), (ii), and (iv) hold with $n=-1$ and $\sigma=\sigma_{0}$.

Assume that there exists a number $n_{*} \in\{-1,0,1,2, \ldots\}$ such that assertions (i), (ii), and (iv) hold with $n=n_{*}$ and $\sigma=\sigma_{n_{*}+1}$. We first prove assertion (i) for $n=n_{*}+1$. Since assertion (i) holds with $n=n_{*}$ and $F_{n}(x, t)=$ $\kappa\left|U_{n}(x, t)\right|^{p-1} U_{n}(x, t)$, we obtain

$$
\sup _{t>0}(1+t)^{A_{p}}\left[t^{(N / 2)(1-1 / q)+1 /(2 q)}\left\|F_{n_{*}}(t)\right\|_{q, \partial \boldsymbol{R}_{+}^{N}}+(1+t)^{-l / 2} t^{1 / 2}\left\|F_{n_{*}}(t)\right\|_{l, \partial \boldsymbol{R}_{+}^{N}}\right]<\infty,
$$

for any $q \in[1, \infty]$ and $l \in[0, K]$. This together with Lemma 3.3 (i) implies that

$$
\sup _{t>0}(1+t)^{A_{p}-l / 2} t^{1 / 2}\left\|Q_{K}(t) \gamma\left[F_{n_{*}}(t)\right]\right\|_{l, \boldsymbol{R}^{N-1}}<\infty
$$

for any $l \in[0, K]$. Since $A_{p}>1 / 2$, by (2.11), (2.14) with $n=0,(3.12)$, and (4.8) we have

$$
\begin{aligned}
\left\|U_{n_{*}+1}(t)\right\| \|_{l} \leq & \left\|U_{0}(t)\right\|\left\|_{l}+\right\| \int_{0}^{t} D\left[Q_{K}(t) \gamma\left[F_{n_{*}}(t)\right]\right](t-s) d s \|_{l} \\
\preceq & (1+t)^{l / 2}+\int_{0}^{t}\left\|Q_{K}(s) \gamma\left[F_{n_{*}}(s)\right]\right\|_{l, \boldsymbol{R}^{N-1}} d s \\
& +\int_{0}^{t}\left(1+(t-s)^{l / 2}\right)\left\|Q_{K}(s) \gamma\left[F_{n_{*}}(s)\right]\right\|_{1, \boldsymbol{R}^{N-1}} d s \\
\preceq & (1+t)^{l / 2}+\int_{0}^{t}(1+s)^{-A_{p}+l / 2} s^{-1 / 2} d s \\
& +\int_{0}^{t}\left(1+(t-s)^{l / 2}\right)(1+s)^{-A_{p}} s^{-1 / 2} d s \preceq(1+t)^{l / 2}
\end{aligned}
$$

for all $t>0$. Furthermore, applying a similar argument as above, we obtain

$$
t^{1 / 2}|| U_{n_{*}+1}(t) \mid \|_{l, \partial \boldsymbol{R}_{+}^{N}}, \quad t>0 . \preceq(1+t)^{l / 2}
$$

On the other hand, by (2.14) with $n=0,(S 3)$, and (3.9) we have

$$
\begin{aligned}
\left|U_{n_{*}+1}(x, t)\right| \leq & \left|U_{0}(x, t)\right|+\int_{0}^{t}\left|D\left[Q_{K}(s) \gamma\left[F_{n_{*}}(s)\right]\right](t-s)\right| d s \\
\preceq & t^{-N / 2}+\int_{0}^{t / 2}(t-s)^{-N / 2}\left\|Q_{K}(s) \gamma\left[F_{n_{*}}(s)\right]\right\|_{1, \boldsymbol{R}^{N-1}} d s \\
& +\int_{t / 2}^{t}(t-s)^{-1 / 2} s^{-(N-1) / 2}\left\|Q_{K}(s) \gamma\left[F_{n_{*}}(s)\right]\right\|_{1, \boldsymbol{R}^{N-1}} d s
\end{aligned}
$$




$$
\begin{aligned}
& \preceq t^{-N / 2}\left(1+\int_{0}^{t / 2}(1+s)^{-A_{p}} s^{-1 / 2} d s+\int_{t / 2}^{t}(t-s)^{-1 / 2}(1+s)^{-A_{p}} d s\right) \\
& \preceq t^{-N / 2}
\end{aligned}
$$

for all $(x, t) \in \overline{\boldsymbol{R}_{+}^{N}} \times(0, \infty)$. This together with (4.9) and (4.10) implies that assertion (i) holds with $n=n_{*}+1$.

Next we prove that assertions (ii) and (iv) hold with $n=n_{*}+1$ and $\sigma=\sigma_{n_{*}+2}$. Since the solution $u$ satisfies (1.3) and (3.25), due to assertion (i) with $n=n_{*}+1$, it suffices to prove that (2.15) and (2.17) hold with $n=n_{*}+1$ and $\sigma=\sigma_{n_{*}+2}$ for all sufficiently large $t$. Put $z(t):=u(t)-U_{n_{*}+1}(t)$. Then, by (2.4), (2.11), and the semigroup properties of $S(t)$ and $e^{t \Delta^{\prime}}$ we have

$$
\begin{aligned}
z(x, t)= & P_{K}(t) u(t)+\sum_{|\alpha| \leq K, \alpha_{N} \notin \mathcal{O}} \int_{0}^{t} S(t-s) N_{\alpha}(\gamma[F(s)], s) g_{\alpha}(s) d s \\
& -2 \int_{0}^{t} G_{1}\left(x_{N}, t-s\right) e^{(t-s) \Delta^{\prime}}\left(\sum_{|\tilde{\alpha}| \leq K} \tilde{M}_{\tilde{\alpha}}(\gamma[F(s)], s) \tilde{g}_{\tilde{\alpha}}(s)+Q_{K}(s) \gamma\left[F_{n_{*}}(s)\right]\right) d s .
\end{aligned}
$$

Then, by Lemma 3.2 (i) we obtain

$$
\begin{cases}\partial_{t} z=\Delta z & \text { in } \boldsymbol{R}_{+}^{N} \times(0, \infty), \\ \partial_{v} z=Q_{K}(t)\left(\gamma\left[\tilde{F}_{n_{*}}(t)\right]\right) & \text { on } \partial \boldsymbol{R}_{+}^{N} \times(0, \infty), \\ z(x, 0)=\left(P_{K}(0) u(0)\right)(x) & \text { in } \boldsymbol{R}_{+}^{N}\end{cases}
$$

where $\tilde{F}_{n}(t)=F(t)-F_{n}(t)$. This implies that

$$
z(t)=S\left(t-t_{0}\right) z\left(t_{0}\right)+\int_{t_{0}}^{t} D\left[Q_{K}(s)\left(\gamma\left[\tilde{F}_{n_{*}}(s)\right]\right)\right] d s
$$

for all $t>t_{0} \geq 0$. Let $q \in[1, \infty]$. By $(S 1)$ we have

$$
\begin{aligned}
t^{(N / 2)(1-1 / q)}\|S(t) z(0)\|_{q} & =t^{(N / 2)(1-1 / q)}\|S(t / 2) S(t / 2) z(0)\|_{q} \\
& \preceq\|S(t / 2) z(0)\|_{1}, \quad t>0 .
\end{aligned}
$$

Similarly, by $(S 2)$ we obtain

$$
\begin{aligned}
& t^{(N / 2)(1-1 / q)+1 /(2 q)}\|S(t) z(0)\|_{q, \partial \boldsymbol{R}_{+}^{N}} \\
& \quad=t^{(N / 2)(1-1 / q)+1 /(2 q)}\|S(t / 2) S(t / 2) z(0)\|_{q, \partial \boldsymbol{R}_{+}^{N}} \\
& \quad \preceq\|S(t / 2) z(0)\|_{1}
\end{aligned}
$$


for all $t>0$. Furthermore, since it follows from (2.8) that

$$
\int_{\boldsymbol{R}_{+}^{N}} x^{\alpha} z(x, 0) d x=\int_{\boldsymbol{R}_{+}^{N}} x^{\alpha}\left(P_{K}(0) u(0)\right)(x) d x=0, \quad|\alpha| \leq K \text { with } \alpha_{N} \notin \mathcal{O},
$$

we can apply Lemma 3.1 (ii) to have

$$
\|S(t / 2) z(0)\|_{1} \preceq t^{-K / 2}, \quad t>0 .
$$

This together with (4.12) and (4.13) implies

$$
t^{(N / 2)(1-1 / q)}\left(\|S(t) z(0)\|_{q}+t^{1 /(2 q)}\|S(t) z(0)\|_{q, \partial \boldsymbol{R}_{+}^{N}} \preceq t^{-K / 2}, \quad t>0 .\right.
$$

On the other hand, by (1.3) and (2.13) with $n=n_{*}$ we have

$$
\left|\tilde{F}_{n_{*}}(x, t)\right| \preceq(1+t)^{-A_{p}}\left|u(x, t)-U_{n_{*}}(x, t)\right|
$$

for all $(x, t) \in \overline{\boldsymbol{R}_{+}^{N}} \times(0, \infty)$. Then, since assertions (ii) and (iv) hold with $n=n_{*}$ and $\sigma=\sigma_{n_{*}+1}$, by (4.15) we obtain

$$
\begin{aligned}
& \sup _{t>0} t^{(N / 2)(1-1 / q)+1 /(2 q)+\mu_{n_{*}+1}}\left\|\tilde{\boldsymbol{F}}_{n_{*}}(t)\right\|_{q, \partial \boldsymbol{R}_{+}^{N}} \\
&\left.\quad+(1+t)^{-l / 2+\mu_{n_{*}+1}} t^{1 / 2}\left\|\tilde{\boldsymbol{F}}_{n_{*}}(t)\right\| \|_{l, \partial \boldsymbol{R}_{+}^{N}}\right)<\infty
\end{aligned}
$$

for any $q \in[1, \infty]$ and $l \in[0, K]$. This together with Lemma 3.3 (i) implies that

$$
\sup _{t>0}(1+t)^{-l / 2+\mu_{n_{*}+1}} t^{1 / 2}\left\|Q_{K}(t) \gamma\left[\tilde{F}_{n_{*}}(t)\right]\right\|_{l, \boldsymbol{R}^{N-1}}<\infty
$$

for any $l \in[0, K]$. Furthermore, by (4.16) we can apply Lemma 3.3 (ii) with $\mu^{\prime}=\mu_{n_{*}+1}$, and obtain

$$
\begin{aligned}
& t^{(N / 2)(1-1 / q)}\left\|\int_{0}^{t} D\left[Q_{K}(s) \gamma\left[\tilde{F}_{n_{*}}(s)\right]\right](t-s) d s\right\|_{q} \\
& \preceq t^{-K / 2} \int_{0}^{t}(1+s)^{K / 2-\mu_{n_{*}+1}} s^{-1 / 2} d s=t^{-K / 2} \int_{0}^{t}(1+s)^{-A_{p}+\sigma_{n_{*}+1}} s^{-1 / 2} d s \\
& \preceq t^{-K / 2}+t^{-K / 2} \int_{1}^{t} s^{-A_{p}+\sigma_{n_{*}+1}-1 / 2} d s \\
& = \begin{cases}t^{-K / 2} & \text { if }\left(n_{*}+2\right)\left(2 A_{p}-1\right)>K, \\
t^{-K / 2} \log t & \text { if }\left(n_{*}+2\right)\left(2 A_{p}-1\right)=K, \\
t^{-\left(n_{*}+2\right)\left(A_{p}-1 / 2\right)} & \text { if }\left(n_{*}+2\right)\left(2 A_{p}-1\right)<K,\end{cases}
\end{aligned}
$$

for all sufficiently large $t$. Similarly, we have 


$$
\begin{aligned}
& t^{(N / 2)(1-1 / q)+1 /(2 q)}\left\|\int_{0}^{t} D\left[Q_{K}(s) \gamma\left[\tilde{F}_{n_{*}}(s)\right]\right](t-s) d s\right\|_{q, \partial \boldsymbol{R}_{+}^{N}} \\
& \preceq t^{-K / 2} \int_{0}^{t}(1+s)^{K / 2-\mu_{n_{*}+1} s^{-1 / 2} d s} \\
& = \begin{cases}t^{-K / 2} & \text { if }\left(n_{*}+2\right)\left(2 A_{p}-1\right)>K, \\
t^{-K / 2} \log t & \text { if }\left(n_{*}+2\right)\left(2 A_{p}-1\right)=K, \\
t^{-\left(n_{*}+2\right)\left(A_{p}-1 / 2\right)} & \text { if }\left(n_{*}+2\right)\left(2 A_{p}-1\right)<K,\end{cases}
\end{aligned}
$$

for all sufficiently large $t$. Therefore we apply (4.14), (4.18), and (4.19) to (4.11) with $t_{0}=0$, and obtain inequality (2.15) with $n=n_{*}+1$ for any sufficiently large $t$. Thus assertion (ii) holds with $n=n_{*}+1$.

On the other hand, for any $l \in[0, K]$, we have

$$
\begin{aligned}
(1+t)^{-l / 2}\left(\left\||| z(t)\left|\left\|_{l}+t^{1 / 2} \mid\right\| z(t)\|\|_{l, \partial \boldsymbol{R}_{+}^{N}}\right)\right.\right. & \\
= & \int_{\boldsymbol{R}_{+}^{N}}\left(\frac{1+|x|}{(1+t)^{1 / 2}}\right)^{l}|z(x, t)| d x+t^{1 / 2} \int_{\partial \boldsymbol{R}_{+}^{N}}\left(\frac{1+|x|}{(1+t)^{1 / 2}}\right)^{l}|z(x, t)| d \sigma \\
\preceq & \int_{\boldsymbol{R}_{+}^{N}}\left[1+\left(\frac{1+|x|}{(1+t)^{1 / 2}}\right)^{K}\right]|z(x, t)| d x \\
& +t^{1 / 2} \int_{\partial \boldsymbol{R}_{+}^{N}}\left[1+\left(\frac{1+|x|}{(1+t)^{1 / 2}}\right)^{K}\right]|z(x, t)| d \sigma \\
= & \|z(t)\|_{1}+t^{1 / 2}\|z(t)\|_{1, \partial \boldsymbol{R}_{+}^{N}} \\
& +(1+t)^{-K / 2}\left(\left|\|z(t)\|_{K}+t^{1 / 2}\right|\|z(t)\|_{K, \partial \boldsymbol{R}_{+}^{N}}, \quad t>0 .\right.
\end{aligned}
$$

Then, by (2.15) with $q=1$ and $n=n_{*}+1$ we see that, if there holds (2.17) with $l=K$, then we have (2.17) for $l \in[0, K]$. Thus it suffices to prove (2.17) with $l=K, n=n_{*}+1$, and $\sigma=\sigma_{n_{*}+2}$. Put $Z(t)=\|z(t)\|_{K}$. By (4.11) we have

$$
Z(2 t) \leq\|\mid S(t) z(t)\|\left\|_{K}+\int_{t}^{2 t}\right\|\left\|D\left[Q_{K}(s) \gamma\left[\tilde{F}_{n_{*}}(s)\right]\right](t-s)\right\| \|_{K} d s, \quad t>0 .
$$

Let $\delta>0$. Then, by (2.15) with $n=n_{*}+1$ and $(S 1)$ we have

$$
\begin{aligned}
\|S(t) z(t)\|_{K} & \leq(1+\delta)\|z(t)\|\left\|_{K}+C_{1}\left(1+t^{K / 2}\right)\right\| z(t) \|_{1} \\
& \leq(1+\delta) Z(t)+C_{2} t^{\sigma_{n_{*}+2}}
\end{aligned}
$$


for all $t \geq 1 / 2$, where $C_{1}$ and $C_{2}$ constants. Furthermore, by (3.12) and (4.17) we obtain

$$
\begin{aligned}
& \int_{t}^{2 t}\left\|D\left[Q_{K}(s) \gamma\left[\tilde{F}_{n_{*}}(s)\right]\right](t-s)\right\|_{K} d s \\
& \preceq \int_{t}^{2 t}\left\|Q_{K}(s) \gamma\left[\tilde{F}_{n_{*}}(s)\right]\right\| \|_{K, \boldsymbol{R}^{N-1}} d s \\
& +\int_{t}^{2 t}\left[1+(2 t-s)^{K / 2}\right]\left\|Q_{K}(s) \gamma\left[\tilde{F}_{n_{*}}(s)\right]\right\|_{1, \boldsymbol{R}^{N-1}} d s \\
& \preceq \int_{t}^{2 t}(1+s)^{K / 2-\mu_{n *+1}} s^{-1 / 2} d s+\int_{t}^{2 t}\left[1+(2 t-s)^{K / 2}\right](1+s)^{-\mu_{n *+1}} s^{-1 / 2} d s \\
& \preceq t^{K / 2-\mu_{n_{*}+1}+1 / 2}=t^{-\left(A_{p}-1 / 2\right)+\sigma_{n_{*}+1}} \preceq t^{\sigma_{n_{*}+2}}, \quad t \geq 1 / 2 .
\end{aligned}
$$

Therefore, by (4.20)-(4.22) we can find a constant $C_{3}$ satisfying

$$
Z(2 t) \leq(1+\delta) Z(t)+C_{3} t^{\sigma_{n *+2}}, \quad t \geq 1 / 2 .
$$

Furthermore, since it follows from (2.14) with $n=n_{*}+1$ and (3.35) that $\sup _{0<t<1} Z(t)<\infty$, applying the same argument as in the proof of [6, Lemma $3.2]$ with the inequality (4.23), we obtain

$$
Z(t) \preceq t^{\sigma_{n_{*}+2}}, \quad t \geq 1 .
$$

On the other hand, by (2.15) with $n=n_{*}+1,(S 2)$, and (4.24) we have

$$
t^{1 / 2} \mid\|S(t) z(t)\|_{K, \partial \boldsymbol{R}_{+}^{N}} \preceq Z(t)+\left(1+t^{K / 2}\right)\|z(t)\|_{1} \preceq t^{\sigma_{n_{*}+2}}, \quad t \geq 1 .
$$

Furthermore, applying a similar argument as in (4.22), we obtain

$$
t^{1 / 2} \int_{t}^{2 t}\left\|\left|D\left[Q_{K}(s) \gamma\left[F(s)-F_{n_{*}}(s)\right]\right](t-s)\right|\right\|_{K, \partial \boldsymbol{R}_{+}^{N}} d s \preceq t^{\sigma_{n_{*}+2}}, \quad t \geq 1 .
$$

This together with (4.11) and (4.25) implies that

$$
t^{1 / 2}\|\mid z(t)\|_{K, \partial \boldsymbol{R}_{+}^{N}} \preceq t^{\sigma_{n_{*}+2}}, \quad t \geq 1 .
$$

By (4.24) and (4.26) we have inequality (2.17) with $n=n_{*}+1$ with $\sigma=\sigma_{n_{*}+2}$ for any sufficiently large $t$. Therefore assertions (ii) and (iv) hold with $n=n_{*}+1$ for all $t>0$. Thus, by induction we see that (2.14), (2.15) and (2.17) hold with $\sigma=\sigma_{n+1}$ for all $n=0,1,2, \ldots$, and assertions (i), (ii), and (iv) of Theorem 2.1 follow.

We complete the proof of Theorem 2.1. 
Proof of Theorem 2.1. It suffices to prove assertion (iii) of Theorem 2.1. Since there holds (2.16) for the case $K>[K]$ by Theorem 2.1 (ii), it suffices to prove (2.16) for the case $K=[K]$. Let $K=[K]$ and $n \in\{0,1,2, \ldots\}$ be such that

$$
(n+1)\left(2 A_{p}-1\right)>K .
$$

Then we can take a positive constant $\sigma$ so that

$$
K-n\left(2 A_{p}-1\right)<2 \sigma<2 A_{p}-1 .
$$

Put $\quad z_{n}(t)=u(t)-U_{n}(t), \quad \tilde{F}_{n}(t)=F(t)-F_{n}(t), \quad$ and $\quad 2 \varepsilon:=2 A_{p}-1-2 \sigma>0$. Then, by (4.27) we apply Theorem 2.1 (ii) and (iv) to obtain

$$
\begin{aligned}
& t^{(N / 2)(1-1 / q)+1 /(2 q)}\left\|\tilde{F}_{n-1}(t)\right\|_{q, \partial \boldsymbol{R}_{+}^{N}}+(1+t)^{-l / 2} t^{1 / 2}\left\|\tilde{\boldsymbol{F}}_{n-1}(t)\right\| \|_{l, \partial \boldsymbol{R}_{+}^{N}} \\
& \quad \preceq(1+t)^{-A_{p}}\left\{t^{(N / 2)(1-1 / q)+1 /(2 q)}\left\|z_{n-1}(t)\right\|_{q, \partial \boldsymbol{R}_{+}^{N}}+(1+t)^{-l / 2} t^{1 / 2}\left\|z_{n-1}(t)\right\| \|_{l, \partial \boldsymbol{R}_{+}^{N}}\right\} \\
& \quad \preceq(1+t)^{-A_{p}}\left[(1+t)^{-K / 2+\sigma}+(1+t)^{-n\left(A_{p}-1 / 2\right)}\right] \\
& \quad \preceq(1+t)^{-A_{p}-K / 2+\sigma} \preceq(1+t)^{-K / 2-1 / 2-\varepsilon}, \quad t>0,
\end{aligned}
$$

where $q \in[1, \infty]$ and $l \in[0, K]$. This together with Lemma 3.3 (i) yields

$$
(1+t)^{-l / 2}\left\|Q_{K}(t) \gamma\left[\tilde{F}_{n-1}(t)\right] \mid\right\|_{l, \boldsymbol{R}^{N-1}} \preceq(1+t)^{-K / 2-1 / 2-\varepsilon} t^{-1 / 2}, \quad t>0 .
$$

By (4.11), for any $L>0$, we have

$$
\begin{aligned}
z_{n}(t) & =S(t) z_{n}(0)+\int_{0}^{t} D\left[Q_{K}(s) \gamma\left[\tilde{F}_{n-1}(s)\right]\right](t-s) d s \\
& =S(t) z_{n}(0)+\left(\int_{t / 2}^{t}+\int_{L}^{t / 2}+\int_{0}^{L}\right) D\left[Q_{K}(s) \gamma\left[\tilde{F}_{n-1}(s)\right]\right](t-s) d s \\
& =: S(t) z_{n}(0)+I_{1}(t)+I_{2}(t)+I_{3}(t), \quad t \geq 2 L .
\end{aligned}
$$

Since $z_{n}(0)=P_{K}(0) u(0)$, by $(2.8)$ we have

$$
\int_{\boldsymbol{R}^{N}} x^{\alpha} z_{n}(0) d x=0, \quad|\alpha| \leq[K]=K \text { with } \alpha_{N} \notin \mathcal{O},
$$

and by $(S 1),(S 2)$, and Lemma 3.1 (ii) we obtain

$$
\begin{aligned}
& \lim _{t \rightarrow \infty} t^{(N / 2)(1-1 / q)+K / 2}\left\|S(t) z_{n}(0)\right\|_{q} \preceq \lim _{t \rightarrow \infty} t^{K / 2}\left\|S(t / 2) z_{n}(0)\right\|_{1}=0, \\
& \lim _{t \rightarrow \infty} t^{(N / 2)(1-1 / q)+1 /(2 q)+K / 2}\left\|S(t) z_{n}(0)\right\|_{q, \partial \boldsymbol{R}_{+}^{N}} \preceq \lim _{t \rightarrow \infty} t^{K / 2}\left\|S(t / 2) z_{n}(0)\right\|_{1}=0 .
\end{aligned}
$$


We first give the estimate for $I_{1}(t)$. By $(S 3)$, (4.28), and Lemma 3.3 (i) we obtain

$$
\begin{aligned}
t^{N / 2}\left|I_{1}(x, t)\right| & \left.\preceq t^{N / 2} \int_{t / 2}^{t}(t-s)^{-1 / 2} s^{-(N-1) / 2} \| Q_{K}(s) \gamma\left[\tilde{\boldsymbol{F}}_{n-1}(s)\right]\right] \|_{1, \partial \boldsymbol{R}_{+}^{N}} d s \\
& \preceq t^{-K / 2-1 / 2-\varepsilon} \int_{t / 2}^{t}(t-s)^{-1 / 2} d s \preceq t^{-K / 2-\varepsilon}
\end{aligned}
$$

for all $(x, t) \in \overline{\boldsymbol{R}_{+}^{N}} \times(0, \infty)$. This implies that

$$
t^{N / 2}\left(\left\|I_{1}(t)\right\|_{\infty}+\left\|I_{1}\right\|_{\infty, \partial \boldsymbol{R}_{+}^{N}}\right)=o\left(t^{-K / 2}\right)
$$

as $t \rightarrow \infty$. Furthermore, by (3.9) and (4.28) with $l=0$ we obtain

$$
\begin{aligned}
\left\|I_{1}(t)\right\|_{1} & \leq \int_{t / 2}^{t}\left\|Q_{K}(s) \gamma\left[\tilde{F}_{n-1}(t)\right]\right\|_{1, \boldsymbol{R}^{N-1}} d s \\
& \preceq \int_{t / 2}^{t} s^{-K / 2-1-\varepsilon} d s \preceq t^{-K / 2-\varepsilon}=o\left(t^{-K / 2}\right)
\end{aligned}
$$

as $t \rightarrow \infty$. Similarly we have

$$
t^{1 / 2}\left\|I_{1}(t)\right\|_{1, \partial \boldsymbol{R}_{+}^{N}} \preceq t^{-K / 2-1 / 2-\varepsilon} \int_{t / 2}^{t}(t-s)^{-1 / 2} d s \preceq t^{-K / 2-\varepsilon}=o\left(t^{-K / 2}\right)
$$

as $t \rightarrow \infty$. This together with (4.31) and (4.32) yields

$$
t^{(N / 2)(1-1 / q)}\left(\left\|I_{1}(t)\right\|_{q}+t^{1 /(2 q)}\left\|I_{1}(t)\right\|_{q, \partial \boldsymbol{R}_{+}^{N}}\right)=o\left(t^{-K / 2}\right) \quad \text { as } t \rightarrow \infty .
$$

Next we give the estimates for $I_{2}(t)$ and $I_{3}(t)$. By Lemma 3.1 (ii), (3.9), (4.28), and the semigroup property of $e^{t \Delta^{\prime}}$ we have

$$
\begin{aligned}
t^{(N / 2)(1-1 / q)}\left(\left\|I_{2}(t)\right\|_{q}+t^{1 /(2 q)}\left\|I_{2}(t)\right\|_{q, \partial \boldsymbol{R}_{+}^{N}}\right) \\
\leq t^{(N / 2)(1-1 / q)} \int_{L}^{t / 2}\left\|D\left[Q_{K}(s) \gamma\left[\tilde{F}_{n-1}(s)\right]\right](t-s)\right\|_{q} d s \\
\quad+t^{(N / 2)(1-1 / q)+1 /(2 q)} \int_{L}^{t / 2}\left\|D\left[Q_{K}(s) \gamma\left[\tilde{\boldsymbol{F}}_{n-1}(s)\right]\right](t-s)\right\|_{q, \partial \boldsymbol{R}_{+}^{N}} d s \\
\preceq \int_{L}^{t / 2}\left\|e^{((t-s) / 2) \Delta^{\prime}} Q_{K}(s) \gamma\left[\tilde{\boldsymbol{F}}_{n-1}(s)\right]\right\|_{1, \boldsymbol{R}^{N-1}} d s \\
\preceq \int_{L}^{t / 2}(t-s)^{-K / 2}\left\|Q_{K}(s) \gamma\left[\tilde{F}_{n-1}(s)\right]\right\|_{K, \boldsymbol{R}^{N-1}} d s \\
\preceq t^{-K / 2} \int_{L}^{t / 2} s^{-1-\varepsilon} d s \preceq t^{-K / 2} L^{-\varepsilon}
\end{aligned}
$$


for all sufficiently large $t$. Similarly we obtain

$$
\begin{aligned}
& t^{(N / 2)(1-1 / q)}\left(\left\|I_{3}(t)\right\|_{q}+t^{1 /(2 q)}\left\|I_{3}(t)\right\|_{q, \partial \boldsymbol{R}_{+}^{N}}\right) \\
& \preceq \int_{0}^{L}\left\|e^{((t-s) / 2) \Delta^{\prime}} Q_{K}(s) \gamma\left[\tilde{F}_{n-1}(s)\right]\right\|_{1, \boldsymbol{R}^{N-1}} d s
\end{aligned}
$$

for all $t>0$. On the other hand, by (2.9) and (4.28) we can apply Lemma 3.1 (ii) to have

$$
\begin{aligned}
& \lim _{t \rightarrow \infty} t^{K / 2}\left\|e^{((t-s) / 2) \Delta^{\prime}} Q_{K}(s) \gamma\left[\tilde{F}_{n-1}(s)\right]\right\|_{1} \\
& =\lim _{t \rightarrow \infty}(t-s)^{K / 2}\left\|e^{((t-s) / 2) \Delta^{\prime}} Q_{K}(s) \gamma\left[\tilde{F}_{n-1}(s)\right]\right\|_{1}=0, \\
& \left\|e^{((t-s) / 2) \Delta^{\prime}} Q_{K}(s) \gamma\left[\tilde{F}_{n-1}(s)\right]\right\|_{1} \preceq(t-s)^{-K / 2}\left\|Q_{K}(s) \gamma\left[\tilde{F}_{n-1}(s)\right]\right\| \|_{K, \boldsymbol{R}^{N-1}} \\
& \preceq t^{-K / 2} s^{-1 / 2}, \quad t \geq 2 L,
\end{aligned}
$$

for all $s \in(0, L)$. Therefore we can apply the Lebesgue dominated convergence theorem to (4.35), and obtain

$$
t^{(N / 2)(1-1 / q)}\left\|I_{3}(t)\right\|_{q}=o\left(t^{-K / 2}\right)
$$

as $t \rightarrow \infty$. Combining (4.29), (4.30), (4.33), (4.34), and (4.36), we see that there exists a constant $C_{4}$ such that

$$
\limsup _{t \rightarrow \infty} t^{(N / 2)(1-1 / q)+K / 2}\left(\left\|z_{n}(t)\right\|_{q}+t^{1 /(2 q)}\|z(t)\|_{q, \partial \boldsymbol{R}_{+}^{N}}\right) \leq C_{4} L^{-\varepsilon} .
$$

Then, since $L$ is arbitrary, we have

$$
\lim _{t \rightarrow \infty} t^{(N / 2)(1-1 / q)+K / 2}\left(\left\|z_{n}(t)\right\|_{q}+t^{1 /(2 q)}\|z(t)\|_{q, \partial \boldsymbol{R}_{+}^{N}}\right)=0 .
$$

Thus we have (2.16) for the case $K=[K]$, and the proof of Theorem 2.1 is complete.

Finally we prove Corollary 2.1.

Proof of Corollary 2.1. We first prove (2.19) with $K \in(0,1)$. Put $\mathscr{F}(s)=$ $\kappa|s|^{p-1} s$. By (1.8) and (2.11) we have

$$
\begin{aligned}
R(x, t)= & u(x, t)-U_{0}(x, t)-\left(M-M_{0}(u(t), t)+\int_{0}^{t} N_{0}(\gamma[\mathscr{F}(u(s))], s) d s\right) g(x, t) \\
& +2 \int_{0}^{t} G_{1}\left(x_{N}, t-s\right) \tilde{M}_{0}(\gamma[\tilde{F}(u(s)), s]) d s \tilde{g}\left(x^{\prime}, t\right)
\end{aligned}
$$


for all $(x, t) \in \overline{\boldsymbol{R}_{+}^{N}} \times(0, \infty)$. This together with (2.5), (2.7), and (2.12) yields

$$
\begin{aligned}
R(x, t)= & u(x, t)-U_{0}(x, t)-\int_{0}^{\infty} \int_{\partial \boldsymbol{R}_{+}^{N}} \mathscr{F}(u(x, s)) d \sigma d s g(x, t) \\
& +2 \int_{0}^{t} G_{1}\left(x_{N}, t-s\right) \int_{\partial \boldsymbol{R}_{+}^{N}} \mathscr{F}(u(x, s)) d \sigma d s \tilde{g}\left(x^{\prime}, t\right) \\
= & u(x, t)-U_{0}(x, t)-\int_{t}^{\infty} \int_{\partial \boldsymbol{R}_{+}^{N}} \mathscr{F}(u(x, s)) d \sigma d s g(x, t) \\
& -2\left(\int_{0}^{t / 2}+\int_{t / 2}^{t}\right)\left(\int_{\partial \boldsymbol{R}_{+}^{N}} \mathscr{F}(u(x, s)) d \sigma\right) \\
& \times\left(G_{1}\left(x_{N}, 1+t\right)-G_{1}\left(x_{N}, t-s\right)\right) d s \tilde{g}\left(x^{\prime}, t\right) \\
= & u(x, t)-U_{0}(x, t)-\int_{t}^{\infty} \int_{\partial \boldsymbol{R}_{+}^{N}} \mathscr{F}(u(x, s)) d \sigma d s g(x, t) \\
& +I_{1}(x, t)+I_{2}(x, t)
\end{aligned}
$$

for all $(x, t) \in \overline{\boldsymbol{R}_{+}^{N}} \times(0, \infty)$. Since it follows from (1.3) that

$$
\|\mathscr{F}(u(s))\|_{1, \partial \boldsymbol{R}_{+}^{N}} \preceq(1+s)^{-A_{p}} s^{-1 / 2}, \quad s>0,
$$

by (3.2) we obtain

$$
\left\|I_{1}(t)\right\|_{1} \preceq \int_{t / 2}^{t}\|\mathscr{F}(u(s))\|_{1, \partial \boldsymbol{R}_{+}^{N}} d s \preceq \int_{t / 2}^{\infty} s^{-A_{p}-1 / 2} d s \preceq t^{-A_{p}+1 / 2}, \quad t>0 .
$$

Similarly it holds

$$
t^{N / 2}\left\|I_{1}(t)\right\|_{\infty} \preceq \int_{t / 2}^{t} s^{-A_{p}-1 / 2} d s+t^{1 / 2} \int_{t / 2}^{t}(t-s)^{-1 / 2} s^{-A_{p}-1 / 2} d s \preceq t^{-A_{p}+1 / 2}, \quad t>0 .
$$

This together with (4.39) implies that

$$
t^{(N / 2)(1-1 / q)}\left\|I_{1}(t)\right\|_{q} \preceq t^{-A_{p}+1 / 2}, \quad t>0,
$$

for any $q \in[1, \infty]$. On the other hand, for any $s \in[0, t / 2]$, applying the mean value theorem, we see that there exists a constant $s_{*} \in(t-s, 1+t) \subset(t / 2,1+t)$ such that

$$
\left|G_{1}\left(x_{N}, 1+t\right)-G_{1}\left(x_{N}, t-s\right)\right| \preceq(1+s)\left|\partial_{t} G_{1}\left(x_{N}, s_{*}\right)\right|, \quad x_{N} \in \boldsymbol{R}_{+} .
$$

This together with (1.3), (3.1), and (3.2) yields

$$
\left\|I_{2}(t)\right\|_{1} \preceq \int_{0}^{t / 2}(1+s)^{-A_{p}+1} s^{-1 / 2} s_{*}^{-1} d s \preceq t^{-1} \int_{0}^{t / 2}(1+s)^{-A_{p}+1} s^{-1 / 2} d s, \quad t>0 .
$$


Similarly we have

$$
\begin{aligned}
t^{N / 2}\left\|I_{2}(t)\right\|_{\infty} & \preceq t^{1 / 2} \int_{0}^{t / 2}(1+s)^{-A_{p}+1} s^{-1 / 2} s_{*}^{-3 / 2} d s \\
& \preceq t^{-1} \int_{0}^{t / 2}(1+s)^{-A_{p}+1} s^{-1 / 2} d s, \quad t>0 .
\end{aligned}
$$

Therefore, for any $q \in[1, \infty]$, we obtain

$$
\begin{aligned}
& t^{(N / 2)(1-1 / q)}\left\|I_{2}(t)\right\|_{q} \preceq t^{-1} \int_{0}^{t / 2}(1+s)^{-A_{p}+1} s^{-1 / 2} d s \\
&= \begin{cases}O\left(t^{-1 / 2}\right) & \text { if } A_{p} \geq 1, \\
O\left(t^{-\left(A_{p}-1 / 2\right)}\right) & \text { if } 1 / 2<A_{p}<1,\end{cases}
\end{aligned}
$$

as $t \rightarrow \infty$. Substituting (2.15) with $n=0,(3.2)$, (4.38), (4.40), and (4.41) to (4.37) we have (2.19) with $K \in(0,1)$. Furthermore, applying a similar argument as in the proof of [9, Proposition 5.1] with (4.3)-(4.6), we obtain (2.19) with $K=1$ and (2.20), and the proof of Corollary 2.1 is complete.

Acknowledgement. The author of this paper was supported in part by the Grant for Basic Science Research Projects from The Sumitomo Foundation (No. 110623) and by the Grant-in-Aid for Young Scientists (B) (No. 24740107) from Japan Society for the Promotion of Science.

\section{References}

[ 1 ] Chlebík, M. and Fila, M., From critical exponents to blow-up rates for parabolic problems, Rend. Mat. Appl. (7), 19 (1999), 449-470.

[2] Chlebík, M. and Fila, M., Some recent results on blow-up on the boundary for the heat equation, Banach Center Publ., 52, Polish Acad. Sci., Warsaw, 2000, pp. 61-71.

[ 3 ] Deng, K., Fila, M. and Levine, H. A., On critical exponents for a system of heat equations coupled in the boundary conditions, Acta Math. Univ. Comenian. (N.S.), 63 (1994), 169-192.

[4] Fila, M., Boundedness of global solutions for the heat equation with nonlinear boundary conditions, Comment. Math. Univ. Carolin., 30 (1989), 479-484.

[5] Galaktionov, V. A. and Levine, H. A., On critical Fujita exponents for heat equations with nonlinear flux conditions on the boundary, Israel J. Math., 94 (1996), 125-146.

[6] Ishige, K., Ishiwata, M. and Kawakami, T., The decay of the solutions for the heat equation with a potential, Indiana Univ. Math. J., 58 (2009), 2673-2708.

[7] Ishige, K. and Kawakami, T., Global solutions of the heat equation with a nonlinear boundary condition, Calc. Var. Partial Differential Equations, 39 (2010), 429-457.

[ 8 ] Ishige, K. and Kawakami, T., Asymptotic expansion of solutions of the Cauchy problem for nonlinear parabolic equations, J. Anal. Math., 121 (2013), 317-351. 
[9] Ishige, K. and Kawakami, T., Refined asymptotic profiles for a semilinear heat equation, Math. Ann., 353 (2012), 161-192.

[10] Kawakami, T., Global existence of solutions for the heat equation with a nonlinear boundary condition, J. Math. Anal. Appl., 368 (2010), 320-329.

[11] Kawakami, T., Entropy dissipation method for the solutions of the heat equation with a nonlinear boundary condition, Adv. Math. Sci. Appl., 20 (2010), 169-192.

[12] Quittner, P. and Souplet, P., Bounds of global solutions of parabolic problems with nonlinear boundary conditions, Indiana Univ. Math. J., 52 (2003), 875-900.

[13] Quittner, P. and Souplet, P., Superlinear Parabolic Problems, Blow-up, Global Existence and Steady States, Birkhäuser Advanced Texts: Basler Lehrbücher, Birkhäuser Verlag, Basel, 2007.

\author{
nuna adreso: \\ Department of Mathematical Sciences \\ Osaka Prefecture University \\ 1-1 Gakuen-cho, Nakaku, Sakai \\ Osaka 599-8531 \\ Japan \\ E-mail: kawakami@ms.osakafu-u.ac.jp
}

(Ricevita la 28-an de aŭgusto, 2012) 